\title{
Mitochondrial permeability transition: a molecular lesion with multiple drug targets
}

\section{Authors}

Thomas Briston ${ }^{1 *}$, David Selwood ${ }^{2}$, Gyorgy Szabadkai3 ${ }^{3,4,5}$, Michael R Duchen ${ }^{3}$

${ }^{1}$ Neurology Innovation Centre, Hatfield Research Laboratories, Eisai Ltd., Hatfield, UK

${ }^{2}$ Wolfson Institute for Biomedical Research, Division of Medicine, University College London, London, UK

${ }^{3}$ Department of Cell and Developmental Biology, Consortium for Mitochondrial Research, University College London, London, UK

${ }^{4}$ Department of Biomedical Sciences, University of Padua, Padua, Italy

5 The Francis Crick Institute, London, UK

* Correspondence: thomas briston@eisai.net (Thomas Briston)

Key words: mitochondria, mPTP, cyclophilin D, drug discovery, calcium

\begin{abstract}
Mitochondrial permeability transition, as the consequence of opening of a mitochondrial permeability transition pore (mPTP), is a cellular catastrophe. Initiating bioenergetic collapse and cell death, it has been implicated in the pathophysiology of major human diseases, including neuromuscular diseases of childhood, ischaemia-reperfusion injury and age related neurodegenerative disease. MPTP represents a major therapeutic target, as opening can be prevented by a number of compounds. However, clinical studies have so far been disappointing. We therefore address the prospects and challenges faced in translating in vitro findings to clinical benefit. We review the role of mPTP opening in disease, discuss recent findings defining the putative structure of mPTP and explore strategies to identify
\end{abstract}


novel, clinically useful mPTP inhibitors, highlighting key considerations in the drug discovery process.

\section{Ruby anniversary: 40 years and finally some success}

Mitochondria are essential for bioenergetic homeostasis and also play a principal role in regulating cellular $\mathrm{Ca}^{2+}$ homeostasis. Mitochondrial $\mathrm{Ca}^{2+}$ overload, especially when combined with oxidative or nitrosative stress and/or ATP depletion, triggers an abrupt increase in the permeability of the inner mitochondrial membrane (IMM) through the opening of a highconductance voltage- and $\mathrm{Ca}^{2+}$ - sensitive channel, known as the mitochondrial permeability transition pore (mPTP) (Figure 1; Key Figure). mPTP opening causes the collapse of mitochondrial membrane potential and bioenergetic failure, coupled with the redistribution of low molecular weight solutes of less than $1500 \mathrm{Da}$ across the IMM and mitochondrial swelling [1, 2].

Approximately 40 years have passed since the first biochemical description of the mitochondrial permeability transition in isolated mitochondria by Haworth and Hunter [3] and 30 years since the discovery that cyclosporin $A(C s A)$ inhibits opening of the mitochondrial permeability transition pore (mPTP) in vitro [4]. Since then, mitochondrial dysfunction and persistent mPTP opening has emerged as a final common pathway to cell death underlying many disease states [5-9]. Specific pharmacological modulation of MPTP opening has long been sought. While the MPTP is clearly a druggable target, the development of inhibitors beyond the prototypical compound, CSA, itself limited by offtarget effects and low therapeutic efficacy (see Glossary), has been slow and disappointing. However, in the last 5 years, multiple groups have identified several novel, chemically distinct small molecule inhibitors of the MPTP, opening avenues for drug discovery and development that will have wide therapeutic potential. Most notably, edaravone (Radicut ${ }^{\mathrm{TM}}$ ), a potent anti-oxidant and inhibitor of the permeability transition has been approved for the treatment of amyotrophic lateral sclerosis (ALS) [10] as well as there being early successes in the treatment of the collagenopathies [11-13]. 


\section{Complex bio-architecture and regulation of the MPTP}

The nature of the MPTP complex has long been debated and as yet no definitive biomolecular configuration has been agreed (Fully reviewed elsewhere [14]). Many candidates for the role of a pore forming inner membrane protein have been proposed, including the adenine nucleotide translocase (ANT) [15], the mitochondrial phosphate carrier (PiC) [16], the metalloprotease spastic paraplegia 7 (SPG7) [17], and either dimers, or an uncoupling channel within the c-ring of the $F_{1} F_{0}-A T P$ synthase $[18,19]$. Outer membrane components, the voltage-dependent anion channel (VDAC) [15] and BCL-2 family members BAK/BAX [20] have further been suggested to play a role as modulators of pore opening. All of these hypotheses have been challenged, and loss- or gain-of function genetic studies have failed to validate each hypothesised candidate [21-24], although some are now understood to play potential regulatory roles $[25,26]$. The only component that is unambiguously involved as a regulator of mPTP opening is the peptidyl prolyl cis-trans isomerase (PPlase), cyclophilin D (CypD, also known genetically as Ppif [Box 1]). Indeed, it is the role of CypD that confers sensitivity of pore opening to CsA, and much of the evidence supporting a role for the pore in disease models is supported by evidence of protection in CypD knockout mice (Table 1). Loss of CypD shifts the threshold for mPTP opening to a higher $\mathrm{Ca}^{2+}$ load, however mitochondria remain capable of permeability transition [27]. Despite this caveat, genetic ablation of CypD in the mouse has proven protective in a remarkable array of disease states in different tissues [27-34], although as yet, these data have failed to translate into clinical benefit (Box 2).

Recent data point to a role for the $\mathrm{F}_{1} \mathrm{~F}_{\mathrm{O}}$-ATP synthase in pore formation. One model suggests that dimerisation and CypD mediated conformational changes of the $F_{1} F_{0}-A T P$ synthase complex give rise to the formation of the $\operatorname{MPTP}[18,35]$. CypD may electrostatically interact with the oligomycin sensitivity conferring protein (OSCP) subunit of the enzyme to potentiate or trigger mPTP opening [18]. This model has been recently refined by details of $\mathrm{pH}$ and $\mathrm{Ca}^{2+}$-mediated regulation of the structure [36, 37], and opposes an alternative model, which suggests that the c-ring of the membrane spanning Fo-component would constitute the pore forming unit without dimer formation [19]. Recent work has cast doubt on the $\mathrm{F}_{1} \mathrm{~F}_{\mathrm{O}}$-ATP synthase hypothesis $[22,23]$. He, Walker and colleagues demonstrated persistent mPTP opening in HAP1 cells selectively devoid of $F_{1} F_{0}-A T P$ synthase subunit $c$ 
(forming the c-ring), subunit b and the OSCP (both components of the peripheral stalk), together suggesting that the proton channel forming membrane components and peripheral stalk of the $F_{1} F_{0}-A T P$ synthase are unlikely to be involved in MPTP formation [22, 23]. Furthermore, molecular dynamic simulations considering c-rings of different size and ion-conducting properties suggest that the c-ring, even if isolated from the $F_{1}$-domain is not the MPTP [38], raising more questions over structural conformation (for detailed reviews of mPTP structure see $[14,39])$.

In the context of drug discovery, the absence of detailed bio-architecture makes traditional 'target-based' approaches (beyond CypD [Box 1]) to hit identification impossible. However, given the robust data set placing unregulated mPTP opening as a key event in major human diseases and evidence supporting a protective role for CypD ablation (Table 1), the accumulation of small-molecule mPTP modulators and modern molecular tool box, although optimistic, it is surely a matter of time before the true channel forming structures are unequivocally defined.

\section{Disease relevance of mPTP opening: Lessons from Ppif ${ }^{-1}$ models}

The physiological role of CypD remains something of a puzzle, as Ppif ${ }^{/-}$mice are born healthy, in litters with correct Mendelian ratios and display increased anxiety and adultonset obesity $[27,28,40]$. Beyond behavioural traits, the lack of significant phenotype suggests that CypD inhibition represents a valid therapeutic strategy to delay mPTP opening with little risk of toxicity or off-target effects. The most significant and robust evidence for a role of CypD and mPTP opening in disease derives from studies of ischaemia-reperfusion injury (see Glossary) in the heart and compelling data further support a role for CypD in the pathology of the Bethlem or Ullrich type muscular dystrophies, consequences of collagen $\mathrm{VI}$ -, delta-sarcoglycan- or the more severe laminin-2-deficiencies [31, 33]. Moreover, single studies suggest a disease-modifying role of CypD following intestinal ulceration and enteropathy associated with chronic use of the non-steroidal anti-inflammatory drug (NSAID) diclofenac [41] and bone loss associated with aging-related osteoporosis [42]. Accumulating evidence suggests that cytoprotection in neurodegenerative disease may be possible through therapeutic modulation of mPTP opening. Loss of CypD conferred neuroprotection across models of neurological disease, including Alzheimer's disease, MS 
and ALS. CypD ablation improved learning and memory in mouse models of Alzheimer's disease expressing mutant amyloid precursor protein (mAPP) [34] and preserved axonal integrity in an experimental allergic encephalomyelitis (EAE) mouse model of MS [30] and delayed onset of ALS and prolonged survival in a mSOD1-G93A mouse model [43]. Together these observations, although supporting a role for mPTP opening in disease, reflect only the consequences of CypD knockout, and therefore must be interpreted as such. Despite these caveats, independent observations in multiple pre-clinical models argue that pharmacological modulation of mPTP opening may be beneficial across a range of important pathologies, most of which lack alternative current treatment options.

\section{Tractability of the MPTP: The search for small molecule inhibitors}

\section{Expression of CypD and mPTP sensitivity across tissues}

mPTP opening has been observed in mitochondria isolated from multiple tissues and species $[44,45]$, suggesting that the phenomenon may be universal across cell types, at least in vitro [46-48]. It has proven much more difficult to assess mPTP opening in mitochondria in situ, and mPTP opening has only recently been described in vivo using tritium-labelled 2deoxyglucose ([3H]DOG) [49], a similar technique pioneered by the Griffiths and Halestrap ex vivo $[6,50]$. Importantly, differences in the $\mathrm{Ca}^{2+}$ threshold for mPTP opening have been described in mitochondria from different tissues $[44,45,51]$. CypD expression appears to be key in determining the sensitivity to stress-induced mPTP opening but correlations between CypD protein expression patterns and tissue specific MPTP formation has not been studied comprehensively. It has been shown that CypD expression is higher in mitochondria isolated from the rat brain substantia nigra compared to striatum and cortex and to be higher in non-synaptic mitochondria and inter-neurones, potentially explaining the heterogeneous response to MPTP formation observed in brain mitochondria and suggesting regional differences in sensitivity [52]. Finally, CypD protein expression and activity may be tightly regulated through complex interactions with hematopoietic-substrate-1 associated protein X-1 (HAX-1) [53], and heat-shock protein-90 [54], further complicating therapeutic targeting. 


\section{Structural considerations}

Considering that the channel forming units of the MPTP complex are not yet definitively identified, structural concerns for therapeutic targeting are limited to CypD. CypD is one of 17 cyclophilins in humans all of which share common architecture (61\% to $86 \%$ homology), whereby two $\alpha$-helices enclose eight antiparallel $\beta$-sheets. Catalytic activity and CsAsensitivity are determined by a tryptophan (Trp-121) within a short $\alpha$-helical turn between loops $\beta 6$ and $\beta 7$ [55]. 11 of the 17 cyclophilins are known to be CsA sensitive. PPlase domains contain two binding pockets (S1' and S2). "Gatekeeper" residues control substrate access to $S 2$ [55]. The S2 pocket is defined by the residues T73, S81, K82, A103, T107, S110 and Q111 in CypA and by TGKATSQ in CypD other cyclophilins show more diversity in the S2 pocket. Structural distinctions between each cyclophilin are also observed between $\beta 1-\beta 2$ and $\alpha 2-\beta 8$ loops outside of the active site and may be involved in modulation of activity via protein-protein interactions (Further reading see [56]). The BioGRID databased indicates 36 binding partners for CypD but whether they all interact with the so-called isomerase site is unknown. Complete elucidation of molecular regulation, signaling and structural characterisation of all cyclophilin family members will ultimately facilitate rational drug design. However, the observed structural diversity, particularly around the S2 pocket and "gatekeeper" regions, presents an attractive strategy with respect to achieving isomerase isoform selectivity, although no tool/reference compounds exist to model this hypothesis in vitro.

\section{Discovery and development of small molecule mPTP inhibitors: difficulties in achieving selectivity and understanding mechanism}

\section{Inhibitors of CypD}

To date, multiple small molecule MPTP inhibitors have demonstrated efficacy in preclinical disease models. However, without complete understanding of the molecular properties, interpretation of pharmacological data is complex, owing to: (1) polypharmacology (see Glossary) of CsA and derivatives; (2) mechanistic liabilities of compounds that desensitise mPTP opening rather than causing true inhibition, and (3) unknown mechanism of action of CypD-independent inhibitors (as discussed below). Instead, Ppif/- (CypD ablation) models afford a cleaner system to corroborate a role for MPTP perturbation in disease. Most mPTP inhibitors target CypD, with the best characterised being CsA (Figure 2A; Table 1). CsA is a 
hydrophobic undecapeptide and non-selective cyclophilin inhibitor and immunosuppressant, binding a highly conserved groove and occluding the cyclophilin PPlase active site $[57,58]$. In pre-clinical models of mPTP opening, CsA demonstrates a narrow effective concentration range, with cardioprotection observed at $0.2 \mu \mathrm{M}$ and benefit lost at concentrations above $0.4 \mu \mathrm{M}$ following substrate-free anoxia studied and in ex vivo 'Langendorff perfused' hearts $[59,60]$. This narrow therapeutic window often confounds pre-clinical studies and will complicate clinical use as these precise concentrations may be hard to achieve (Box 2). N-methyl-isoleucine-4-cyclosporin (NIM-811) and N-methyl-Dalanine-3-N-ethyl-valine-4-cyclosporin (Debio025/alisporivir and previously known as Unil025) are non-immunosuppressive, semi-synthetic analogues of CsA which lack the calcineurin binding domain $[61,62]$. Both are potent, non-selective cyclophilin inhibitors and have demonstrated activity in multiple in vivo models of myopathy, including dystrophin mutant sapje zebrafish [13], ColVI mutant zebrafish [63], myopathic Col6a1\%, dystrophic $m d x$ mice [64-66] as well as in vitro patient muscle-derived cells [13].

Sanglifehrin $\mathrm{A}(\mathrm{Sf} A)$ is a large molecular weight natural product, isolated from streptomyces sp., and is also a non-selective inhibitor of cyclophilins (Figure 2B), binding both CypD and CypA, and has significant immunosuppressive activity [67]. SfA is structurally unrelated to CsA and but also binds to the isomerase cleft [67]. SfA, CsA and analogues are limited by poor physicochemical properties (aqueous solubility, high molecular weight), polypharmacology and cytotoxicity (most notably nephrotoxicity). All are large molecules $(C s A=\sim 1200 \mathrm{Da})$, with poor blood brain barrier (BBB; see Glossary) permeability, restricting their utility as neuroprotective agents in CNS disease and their therapeutic development potential for treating primary mitochondrial diseases.

A number of groups have identified non-peptidic small molecule inhibitors of CypD [68-70] (Table 1). In silico modelling and in vitro surface plasmon resonance (SPR) identified C-9, a 4aminobenzenesulfonamide derivative, as binding CypD with high affinity (see Glossary) [70, 71]. C-9 inhibits CypD PPlase activity and delays $A \beta$ - and $\mathrm{Ca}^{2+}$-induced mitochondrial swelling and A $\beta$-induced neuronal cell death [71]. Similarly, Shore et al. developed ureabased small molecule, active-site binding CypD inhibitors [68]. Compound 19 protected isolated mouse pancreatic acinar cells from necrotic cell death induced by taurolithocholate 
acid 3-sulfate [68] and demonstrated cytoprotection in models of UV-induced retinal degeneration, reducing oxidative damage and cytochrome c release [72].

Park et al. undertook a novel approach to hit identification, having generated an energybased pharmacophore map based on the co-crystal structure of the CypD-CsA complex [69]. Hits were optimised using in silico pharmacokinetic analysis and molecular docking, and following biological validation and SPR, 2 compounds were identified with a $K_{D}$ in the nanomolar range, which may serve as lead molecules for further development [69]. Biological and technical limitations of the PPlase activity assay, including use of non-natural substrate, speed of reaction, high spontaneous substrate turnover and potential for identifying inhibitors of chymotrypsin inhibitors, make large scale screening challenging and necessitate that binding affinity and the functional consequences must be measured in order to assess activity, and PPlase assays must therefore be complemented by alternative approaches, including assessment of binding using SPR, fluorescence polarisation, homogeneous time resolved fluorescence (HTRF), isothermal calorimetry (ITC) . Furthermore, CypD inhibitors shift the threshold for mPTP opening to a higher $\mathrm{Ca}^{2+}$ load, but do not completely suppress PTP opening [27]. Therefore, the development of mPTP inhibitors with a mechanism distinct from CypD inhibition is attractive.

\section{CypD-independent inhibitors of mPTP opening}

The first identified CypD-independent inhibitor, TRO-40303 (3,5-seco-4-nor-cholestan-5-one oxime-3-ol), binds to the cholesterol binding site of the $18 \mathrm{kDa}$ outer mitochondrial membrane translocator (TSPO; Figure 1; Key Figure; Table 1). Schaller et al. demonstrated that the TSPO ligand TRO-40303 delays oxidative stress-induced mPTP opening in vitro and following in vivo reperfusion injury [73]. However, in experiments using conditional Tspo knockout mice, Šileikytè et al. concluded that the TSPO had no role in mPTP formation, regulation or mPTP-mediated cardiac cell death [74], casting doubt on the prospect for TSPO ligands as a therapeutic paradigm for mPTP modulators. However, despite these data, TRO40303 progressed to Phase II clinical trial, and maybe predictably, yielded disappointing results (Box 2).

A number of other compounds, including derivatives of $\mathrm{N}$-phenyl-benzamides, cinnamic anilides, isoxazoles, PP11 analogues and the small molecule ER-000444793 (Table 1), have 
been identified as novel CypD-independent inhibitors of mPTP opening. (E)-3-(4-fluoro-3hydroxy-phenyl)-N-naphthalen-1-yl-acrylamide, a cinnamic anilide, with potency greater than CsA, inhibits $\mathrm{Ca}^{2+}{ }_{-}$, oxidative stress- and chemical cross-linker-induced mPTP opening [75]. From the same cinnamic anilide series, GNX-4975 has been proposed to bind the interface and stabilise the interaction between PiC and c-conformation of ANT [76], one model hypothesis for mPTP formation and providing the first details on potential compound mechanism [77]. Notably, F is able to cross the blood-brain barrier and has demonstrated efficacy in neurodegenerative disease models, slowing disease progression and increasing lifespan in a mouse model of motor neurone disease (ALS) expressing the G37R-human mutant superoxide dismutase (SOD)-1 [78].

Screening of 363,827 molecules from the NIH Molecular Libraries Small Molecule Repository in a mitochondrial $\mathrm{Ca}^{2+}$-induced swelling assay identified the isoxazole chemotype as a potential 'hit'. Structure activity relationship (SAR; see Glossary) around the scaffold, identified $\mathrm{N}$-(3-chloro-2-methylphenyl)-5-(4-fluoro-3-hydroxyphenyl)isoxazole-3carboxamide (compound 60), with equipotent inhibition to CSA in models of $\mathrm{Ca}^{2+}$-and chemical-induced mPTP opening, cross-species activity and improvements in muscle function and structure in congenital muscular dystrophy associated with a collagen VI mutation in a zebrafish model [12]. The isoxazole ML 404 (CID 72199308) was also identified through the NIH Molecular Libraries Program as a highly potent inhibitor of $\mathrm{Ca}^{2+}$-induced mPTP opening [79]. More recently, 3-(benzyloxy)-5-chloro- $N$-(4-(piperidin-1ylmethyl)phenyl)benzamide (Compound 4), was identified following SAR studies based on the a $\mathrm{N}$-phenyl-benzamide scaffold [80]. Compound 4 delays $\mathrm{Ca}^{2+}$ - and oxidative stressinduced mPTP opening in isolated mitochondria, has favourable physicochemical properties, and the potential for blood-brain barrier and CNS penetration, however no biological validation in cell or animal models have been reported [80]. Compound 4 appears to target directly the MPTP, since it equally inhibits pore opening induced by $\mathrm{Ca}^{2+}$ and redox-sensitive thiol oxidation in the matrix or membranes. Moreover, its inhibitory effect on respiration and toxicity at higher micromolar concentrations indicates that it might target the OXPHOS machinery, and since the effect of oligomycin is not additive to that of Compound 4 , it hints to the involvement of the ATP synthase. Finally, $\mathrm{N}$-(2-benzylphenyl)-2-oxo-1H-quinoline-4carboxamide (ER-000444793) was identified from a screen of 50,000 compounds which 
delayed $\mathrm{Ca}^{2+}$-induced mPTP opening, independent of CypD binding and PPlase inhibition (Table 1) [81]. No further information on the potential target of ER-000444793 is known yet, although its effect on ATP synthesis has been ruled out.

A number of alternative approaches for MPTP inhibition have been reported, probably acting via inhibiting pathways upstream to MPTP opening, including modulation of mitochondrial redox state $[10,82,83]$, ETC function [84] and $\mathrm{Ca}^{2+}$ influx (REF). The most significant achievement to date is the approval of Edaravone (Radicut ${ }^{\mathrm{TM}}$ ) [10] (Box 2), a potent anti-oxidant and inhibitor of MPTP opening and having demonstrated protection in multiple models $[85,86]$. The mitochondrial targeted antioxidant (AntiOxBEN 3 ), targeted to mitochondria by conjugation with triphenylphosphonium $\left(\mathrm{TPP}^{+}\right)$, delayed $\mathrm{Ca}^{2+}$-induced mPTP opening and protected against iron- and hydrogen peroxide-induced cytotoxicity [82]. A small molecule CypD-independent mPTP inhibitor DS44170716 has been described, which apparently reduces mitochondrial $\mathrm{Ca}^{2+}$ uptake but this appears to result from a loss of mitochondrial membrane potential and inhibition of respiration, raising safety concerns and significantly reducing therapeutic potential [84]. DS16570511 has been proposed as a novel inhibitor of the MCU, potentially inhibiting mPTP opening via inhibition of $\mathrm{Ca}^{2+}$ uptake [84]. Finally, screening of the Hoffmann-La Roche compound collection identified Ro-68-3400 as a potent MPTP inhibitor and as binding to VDAC1 [87], however these data have been questioned [88].

Although the mechanism of action is yet to be fully elucidated, all compounds discussed here inhibit mPTP opening through mechanisms distinct from CypD inhibition, together avoiding off-target effects on other cyclophilins and immunomodulation. On-target toxicity is unknown given the absence of a biomolecular target, however many have demonstrated a robust safety profile in vitro. Notwithstanding the recent data supporting a role for $\mathrm{F}_{1} \mathrm{~F}_{\mathrm{O}^{-}}$ ATP synthase in mPTP formation, compound 60, PP11 analogues (compound 10) and ER000444793 are free of potentially detrimental effects on ATP synthesis. Altogether, welldefined HTS campaigns and a sensible critical path for compound discovery and development have together identified a chemically diverse collection of tool/reference molecules that are not restricted by mechanistic limitations of CypD inhibition. These compounds will initially provide chemically useful pharmacophores and help in understanding the exact bio-molecular architecture and regulation of the MPTP complex. 
Lead optimisation iterations may improve potency and pharmacokinetic properties and potentially begin to pave the way to achieving greater therapeutic efficacy.

\section{Strategies for the discovery of small molecule mPTP inhibitors}

\section{Target Validation, hit identification and delineation of a screening flow}

Target Validation (TV; see Glossary) is the initial and most important stage of the drug discovery process and aims to establish whether a biomolecule (protein, nucleic acid) is worthy of significant investment and development. A high-throughput screen (HTS) (see Glossary) may or may not form part of initial TV. However, establishment and optimisation of a HTS compatible screening system as well as delineation and establishment of downstream/orthogonal bioassays (see Glossary) comprising the screening flow and critical path for hit identification are essential for any small molecule screening campaign (Figure 2C). The complex and uncertain nature of the MPTP complex restricts target-based screening strategies, as beyond CypD, there is still an uncertainty about 'target' biomolecules. Instead, phenotypic screening methodologies, using system-based bioassays must be adopted. Phenotypic screening is mechanism of action- and target-agnostic and instead looks to identify small molecule or biological modifiers of a cellular process. The choice of primary screening bioassay is determined by quality (reproducibility, variability and assay window (see glossary)) and can be calculated using a simple statistical measure, the Z-prime score (see Glossary) [89].

A trio of system-based bioassays, each assessing $\mathrm{Ca}^{2+}$-induced mPTP opening in isolated rodent liver mitochondria comprise the most widely used phenotypic screening strategy to address mPTP opening (Figure 2D-F). Delay in mPTP opening defines an active molecule, or 'hit' compound, however, compound-mediated depolarisation of the mitochondrial inner membrane, inhibition of the mitochondrial $\mathrm{Ca}^{2+}$ uniporter (MCU; see Glossary) or of the phosphate carrier $(\mathrm{PiC})$ would each prevent electrophoretic $\mathrm{Ca}^{2+}$ uptake and $\mathrm{Ca}^{2+}$ sequestration, producing data consistent with inhibition of mitochondrial $\mathrm{Ca}^{2+}$-induced swelling (Box 3). To eliminate false positives, compounds are ideally profiled across three mPTP system-based assays in parallel or sequentially (Figure 2D-F and Box 3 ), and the data used to define the mechanism and these strategies have been used successfully to identify mPTP inhibitors, such as ER-000444973 [81]. Furthermore, together or individually defined 
bioassays are used to delineate pharmacology, guide SAR and confirm cross species activity (using mitochondria isolated from human cell lines) (Figure $X$ and Box 3 ).

\section{Challenges in discovering and developing MPTP inhibitors}

\section{Pre-clinical translation}

Lack of efficacy is the most significant reason for clinical failure of a candidate molecule, potentially stemming from poor translation of pre-clinical conclusions. A fundamental determinant for a successful phenotypic screen is the degree to which the bioassay reflects, and is able to predict, success in modulating the (patho-)physiological end-point of interest. Moffat et al, termed 'chain of translatability' to describe a shared mechanistic basis for the bioassay end-point and disease phenotype, qualities which are necessary if they are to have robust predictive power [90]. Given disappointing outcomes from CsA clinical trials (Box 2), pre-clinical models of reperfusion injury have failed to translate [91], leaving the problem of how to assess the clinical utility of MPTP inhibitors. Phenotypic screening approaches used for small molecule mPTP modulators are; (1) outside of the cellular context (i.e. in isolated mitochondria) and (2) do not reflect mechanisms underlying disease pathophysiology (i.e. not causal), therefore together decreasing the probability of successful translation. Given these observations, it will be necessary to have robust secondary bioassays to profile compounds in models with physiological relevance. E.g. an intact cell based assay, allowing specific activation of the MPTP, could further increase the breadth of available compounds acting not only the mPTP but also on its consequences on cell survival, a further step for translatability. Although a variety of cell based mPTP assays have been proposed, the lack of their robustness and specificity so far impeded their routine use for large scale compound screens. Furthermore, target identification following a phenotypic screen is challenging and hence, without clear target deconvolution it is difficult to predict tissue specific activity (based on protein expression) and safety (based on protein function). Further studies on putative pore components are needed in order to introduce single protein target based approaches above CypD. 


\section{Physiological function of the mPTP}

Although mPTP opening is well established as a mechanism of cell death, transient, reversible opening has been linked to numerous physiological processes, including: (1) energy metabolism, (2) $\mathrm{Ca}^{2+}$ and redox signalling and (3) development [92-94]. The ATP synthasome, composed of $\mathrm{F}_{1} \mathrm{~F}_{\mathrm{O}}$-ATP synthase, $\mathrm{PiC}$ and ANT, is an hetero-oligomeric, mitochondrial inner membrane super-assembly [95]. Evidence supports a role for each element of the complex in the formation or regulation of pore opening $[18,25,26]$ and CypD binding $[15,16,96]$. Therefore it is plausible that physical and functional coupling of mPTP components may regulate mitochondrial metabolism [92] and observations suggest that CypD may control F Fo-ATP synthase plasticity; its ability to oligomerise into synthasomes and to respond appropriately to bioenergetic demand [97].

Mitochondria are a central hub for cellular $\mathrm{Ca}^{2+}$ homeostasis [98] and take up $\mathrm{Ca}^{2+}$ from the cytosol during physiological $\mathrm{Ca}^{2+}$ signalling. It has been suggested that low-conductance MPTP opening and partial transmembrane depolarisation may contribute to mitochondrial $\mathrm{Ca}^{2+}$ efflux [93], although these observations have recently been questioned [99]. Furthermore, CypD ablation increases mitochondrial matrix $\mathrm{Ca}^{2+}$, reprogramming cardiac metabolism, consistent with increased activity of $\mathrm{Ca}^{2+}$-dependent dehydrogenases of the Krebs cycle [100].

During development, myocyte differentiation and maturation of mitochondrial structure and function are favored by MPTP closure. Indeed, myocyte differentiation can be pharmacologically and genetically accelerated through MPTP inhibition via regulation of ROS [94]. The physiological role for MPTP opening requires further investigation. However emerging data suggests that therapeutic targeting MPTP opening has to be carefully considered given accumulating evidence of a role in physiology (Box 1 ).

\section{Opportunities for achieving selectivity and efficacy of mPTP inhibitors}

To achieve selectivity for CypD, over distinct cyclophilins, Malouitre et al. took advantage of the fact that CypD is the only mitochondrially localised cyclophilin [101]. Mitochondrial targeting of CsA was achieved by conjugation of a lipophilic TPP ${ }^{+}$cation at the sarcosine-3 position of the CsA ring. Theoretically, accumulation of a compound into mitochondria in 
response to the transmembrane potential is expected to increase matrix concentration of the compound by up to a thousand-fold, reducing the dose required to reach the target and so reducing off-target effects. Indeed, transmembrane potential-driven electrophoretic uptake and matrix accumulation of mitochondrially-targeted CsA (mtCsA) decreased calcineurin binding demonstrated good selectivity for CypD over CypA and induced protection in a model of oxygen and glucose deprivation-induced necrosis in hippocampal neurones $[57,101,102]$, suggesting pharmacological activity remains following substitution.

JW47 is a next generation mitochondrially targeted CsA and features a quinolinium cation in the place of $\mathrm{TPP}^{+}$, removing $\mathrm{TPP}^{+}$-induced toxicity and physicochemical restrictions. JW47 demonstrates less immunosuppressive activity compared to CsA and neuroprotection in a relapsing-progressive EAE model of MS [103]. In vivo efficacy of JW47 provides proof-ofprincipal for the selective targeting of CypD and use of a quinolinium cation as a mitochondrially targeting group. However, to date achieving small molecule, non-artificially targeted selectivity for CypD remains elusive.

Numerous novel biomolecule-targeting modalities have been described which may have application and benefit in achieving cyclophilin specificity and potency. PROteolysis TArgeting Chimeras (PROTACs) are bi-functional molecules that simultaneously recruit an E3-ubiquitin ligase and bind a target protein. PROTACs are composed of 2 distinct ligands, one to bind the target protein and one to recruit E3-ubiqutin ligase, separated by a linker. Forced proximity between the E3-ligase and exposed lysine residues on the bound target protein, drives ubiquitination and proteasomal targeting for degradation [104]. PROTACs are required only to bind the target molecule and need not inhibit the active site or prevent protein-protein interactions, providing an advantage of traditional small molecules, however may have limitations with respect to CNS penetration. Identification of targetbinding moieties has been aided by development of DNA encoded libraries (see Glossary), hugely increasing chemical diversity and aiding ligand identification. Significant work has been done since conception, including applying click chemistry to improve cellular permeability, solubility and pharmacokinetics (CLIPTACs) [105]. It is now conceivable that targeted protein degradation may offer a novel strategy to target traditionally un-druggable proteins and limit protein aggregation. 
RNA targeted therapeutics describes the use of oligonucleotides to modulate target biomolecule expression [106]. One example, single-stranded anti-sense oligonucleotides (ASOs) are chemically modified oligonucleotides of 18-20 nucleotides, which bind their cognate RNA sequence through Watson-Crick hybridization. Occupancy only- (inhibition of translation, steric interference) or occupancy-mediated degradation- (RNase H1 degradation of ASO-RNA complex) mechanisms reduce target protein expression and the technology is now optimised with improve stability, delivery and affinity [106]. Much like PROTACs, substantial target specificity can be achieved, as ASOs can be designed to regions of nonsequence homology. PROTACs and ASOs may provide opportunities to drug the undruggable, increasing specificity, reducing off-target effects and poly-pharmacology associated with traditional small molecules and potentially provides mechanisms to achieve cyclophilin specificity. While CypD is the only unambiguous mPTP regulator, these approaches will undoubtable have utility once further regulators or pore forming units are identified.

\section{Conclusions and Future perspectives}

Despite lack of supporting genetic data linking Ppif perturbation with disease, evidence for a role of the MPTP as a final common path to cell death in a number of major human diseases is extremely compelling [5-9]. Loss-of-function of CypD is likely beneficial and so unlikely to be identified from genome wide studies. MPTP appears to play a major part in the pathophysiology of diseases for which current therapeutic approaches are very limited and inadequate - MS, AD, PD, stroke, ischaemia-reperfusion injury in the heart, brain and kidney and hereditary neuromuscular diseases of childhood together representing an astonishing human and financial burden. It surely follows inevitably that finding a pharmacological solution could have an astonishing impact on healthcare and fulfil a huge unmet clinical need. These considerations provide very strong strategic and commercial arguments to devote significant effort in the search for an effective pharmaceutical solution to target the pore. That this has proven so difficult remains a puzzle, as does the difficulty in establishing the molecular identity of the pore itself (see Outstanding Questions), but both are hopefully just a matter of time. 


\section{Disclosures}

T.B is an employee of Eisai Ltd.

\section{Text Boxes}

\section{Box 1: Cyclophilin D: the only accepted positive regulator of $\mathrm{MPTP}$ opening} An abundance of pharmacological and genetic evidence recognises cyclophilin D (CypD) as the only, universally accepted protein modifier and key positive regulator of mPTP opening [27]. CypD (encoded by the Ppif gene), a mitochondrial matrix localised 21kDa cyclophilin, catalyses the cis-trans isomerisation of peptidyl-prolyl bonds in target proteins [107]. CypD PPlase enzymatic activity is necessary for positive regulation of pore opening [27], abolition of which affords the mechanism for CsA-mediated mPTP inhibition [4]. Genetic or pharmacological ablation of CypD increases the threshold for $\mathrm{Ca}^{2+}$ - and ROS-induced mPTP opening $[27,28]$. However, at $\mathrm{Ca}^{2+}$ concentrations greater than the physiological threshold, despite CypD inhibition, pore opening can still proceed, suggesting a de-sensitising, regulatory role of CypD and not that of a structural component.

CypD has been observed to bind multiple putative MPTP components, including VDAC and ANT [15, 108], PiC [16] and SPG7 [17]. Furthermore, regulatory, non-structural interactions implicate CypD as a signalling hub between multiple functionally distinct proteins and potential mPTP modulators, including p53 [109], complement C1Q binding protein (C1QBP) [110], amyloid beta (AB) [9] and mitochondrial chaperones [54]. Furthermore, CypD has been identified in mitochondrial-associated membrane (MAM) fractions, within a VDAC1/Grp75/IP3R complex [111] (Figure 1; Key Figure). Recent models suggest a functional interaction between CypD and the lateral stalk of the F Fo-ATP synthase, whereby CypD binding decreases enzymatic activity of the ATP synthase complex, in an event favoured by inorganic phosphate (Pi) and inhibited by CsA [96]. The oligomycin-sensitivity conferring protein (OSCP) subunit of the $\mathrm{F}_{1} \mathrm{~F}_{0}-\mathrm{ATP}$ synthase complex has been proposed as the CypD binding site [18, 112], however these data have recently been challenged [22]. CypD is also post-translationally modified, with S-nitrosylation [113] , phosphorylation [114] and acetylation [115] having been proposed to modulate CypD activity. Global knockout of the mitochondrial $\mathrm{Ca}^{2+}$ uniporter (MCU), increases the phosphorylation of $\mathrm{CypD}$ and its 
association with the $\mathrm{F}_{1} \mathrm{~F}_{\mathrm{O}}$-ATP synthase, reducing $\mathrm{Ca}^{2+}$ retention capacity and contributing to the lack of cardioprotection observed following ischemia-reperfusion injury [116]. The complex interactome and post-translational modifications (PTMs) of CypD necessitate further studies, however given the clear relationship between CypD ablation and increased tolerance to mitochondrial stressors, CypD modulation, either via direct inhibition or regulating protein-protein interaction and PTMs represents a valid therapeutic strategy.

\section{Box 2: mPTP inhibitors in the clinic}

Inconsistencies in CsA trials: A number of small clinical studies have explored the efficacy of CsA in improving cardiac outcomes following myocardial infarction-induced reperfusion injury. The first, small pilot phase II study and limited follow up in patients randomised to CsA treatment at time of $\mathrm{PCl}$, identified significantly reduced infarct size during and improvements in cardiac functions $[117,118]$. A number of small trials since, have also identified benefit in CSA treatment in post-operative myocardial injury [119, 120], however others concluded there are no clinical benefits of pre-thrombolytic CsA administration [121]. A larger phase II open label study, the CYCLE (CYCLosporinE A in Reperfused Acute Myocardial Infarction) trial, also revealed no significant changes in primary or biochemical secondary end-points following CsA treatment up to 6 months follow up [122]. Larger phase III trials, such as CIRCUS (Cyclosporine to ImpRove Clinical oUtcome in STEMI patients) observed no significant difference in primary or secondary end-points, including all-cause mortality, ST elevation and infarct size [91], although long coronary occlusion times, high blood glucose at admission, and pharmacological limitations associated with CsA may go some way to explaining clinical failings [123]. However consistent with previous studies, nor could CsA reduce acute renal injury following CABG surgery in the CiPRICS (Cyclosporine to Protect Renal Function in Cardiac Surgery) study [124].

The disappointment of TR040303: The MITOCARE study demonstrated no effect of TRO400303 in limiting myocardial injury following reperfusion between treatment and placebo [125] or effecting pro- inflammatory cytokines at 72 hours following $\mathrm{PCl}$ [126]. These data may have been predicted given data dismissing a role for TSPO in MPTP formation [74]. The history of mPTP inhibitors in the clinic has been disappointing. 
Inconsistencies in trial design, notably the point of compound administration, indication, pharmacokinetic and pharmacodynamic properties polypharmacology of CsA and poor understanding of TSPO biology, may potentially reconcile heterogeneity in clinical measures $[127,128]$.

Clinical success of edaravone: The first phase III studies edaravone demonstrated reduction of revised ALS functional rating scale (ALSFRS-R) over placebo group and efficacy in ALS, however only in early-stage disease [10]. These data were confirmed in a second randomized, double-blinded, placebo-controlled trial [129]. Based on these successful outcomes in 2015 edaravone was approved for ALS in Japan in 2015. Indeed, the success of edaravone and data linking persistent mPTP opening as a key pathophysiological mechanism in cell death across multiple disease states, including neurodegenerative diseases $[9,34,69$, $78,103]$ and muscular dystrophies $[7,11,130]$, opens the door to targeting distinct clinical indications.

\section{Box 3: Assessment of mPTP opening in vitro using isolated mitochondria}

1. MPTP detection by measurement of mitochondrial transmembrane potential: The mitochondrial transmembrane potential is the electric component of the proton motive force [131], measured as voltage differential between the net positively charged, acidic mitochondrial matrix and the inter membrane space (IMS). Serial reduction of complexes I, III and IV of the electron transport chain (ETC), generates energy to pump protons from the matrix, across the inner mitochondrial membrane, to the IMS, generating an electrochemical gradient. Protons pass back down this gradient, through the $\mathrm{F}_{1} \mathrm{Fo}_{\mathrm{O}} \mathrm{ATP}$ synthase (complex V), in a mechanism coupled to ADP phosphorylation.

Tetramethylrhodamine methylester (TMRM) is a fluorescent, cationic and lipophilic dye. TMRM, according to the negative potential of the IMS, is partitioned largely to the mitochondrial matrix, due its positive charge. Mitochondrial accumulation is strictly dependent on mitochondrial inner membrane potential and at low concentration $([T M R M]$ extracellular $=1-20 \mathrm{nM})$, generates fluorescence proportional to the voltage gradient across the IMM [131]. Under these conditions (called TMRM redistribution mode), loss of inner membrane potential, as a 
consequence of ETC inhibition, ETC uncoupling or decreased supply of reducing equivalents, yields a decrease in fluorescence (Figure IA). At high concentration, ([TMRM]extracellular $\geq 100 \mathrm{nM}$; determined empirically for different cell types), fluorescence of mitochondrially localised dye is quenched. Under these conditions, any disruptions to the transmembrane potential yields an increase in fluorescence as quenching is relieved as dye escapes the mitochondria (Figure IB), a process known as de-quenching [132]. Thus, using TMRM redistribution, mPTP will be detected as loss of mitochondrial TMRM fluorescence, while in de-quenching mode a transient increase of TMRM fluorescence indicated MPTP formation.

2. In vitro assessment of mPTP opening using isolated mitochondria: Mitochondrial $\mathrm{Ca}^{2+}$ uptake is transmembrane potential dependent, and under physiological conditions mitochondria can accumulate a large amount of $\mathrm{Ca}^{2+}$ without exceeding the threshold for mPTP opening, due to $\mathrm{Ca}^{2+}$ buffering in the matrix [133]. At extremes of cellular $\mathrm{Ca}^{2+}$ increases, mitochondrial buffering capacity is exceeded and the MPTP opens.

a. $\mathrm{Ca}^{2+}$-induced mitochondrial swelling: Following MPTP opening, mitochondria become swollen due to disruptions in ionic and solute balance. Swollen mitochondria show reduced absorbance at $520-540 \mathrm{~nm}$ as measured by spectrometry. mPTP inhibitors delay the observed decrease in absorbance.

b. Mitochondrial $\mathrm{Ca}^{2+}$ retention capacity: Electrophoretic mitochondrial $\mathrm{Ca}^{2+}$ uptake is mediated through the mitochondrial calcium uniporter (MCU). Repeated addition of small concentrations of $\mathrm{Ca}^{2+}$ to a mitochondrial suspension containing a mitochondrially impermeable, low affinity $\mathrm{Ca}^{2+}$ dye (i.e. Fluo-4FF pentapotassium salt) provides a sensitive measure of mitochondrial $\mathrm{Ca}^{2+}$ uptake and propensity to undergo mPTP opening. MPTP inhibitors allow mitochondria to retain greater amount of $\mathrm{Ca}^{2+}$.

c. $\mathrm{Ca}^{2+}$-induced transmembrane depolarisation: Mitochondrial $\mathrm{Ca}^{2+}$ uptake is transmembrane potential dependent. Excess mitochondrial matrix $\mathrm{Ca}^{2+}$ disrupts the ionic gradient and causes MPTP opening and mitochondrial transmembrane depolarisation. $\mathrm{Ca}^{2+}$-induced redistribution or de-quenching of TMRM is measured using a fluorescence spectrometry. mPTP inhibitors 
delay the $\mathrm{Ca}^{2+}$-induced changes in TMRM fluorescence. Furthermore, measurement of TMRM fluorescence prior to $\mathrm{Ca}^{2+}$ addition identifies compound-induced mitochondrial toxicity.

\section{References}

1. Szabo, I. and M. Zoratti, The mitochondrial megachannel is the permeability transition pore. Journal of bioenergetics and biomembranes, 1992. 24: p. 111-117.

2. Bernardi, P., Mitochondrial transport of cations: channels, exchangers, and permeability transition. Physiol Rev, 1999. 79(4): p. 1127-55.

3. Haworth, R.A. and D.R. Hunter, The Ca2+-induced membrane transition in mitochondria. II. Nature of the Ca2+ trigger site. Archives of biochemistry and biophysics, 1979. 195: p. 460467.

4. Crompton, M., H. Ellinger, and A. Costi, Inhibition by cyclosporin A of a Ca2t-dependent pore in heart mitochondria activated by inorganic phosphate and oxidative stress. The Biochemical journal, 1988. 255: p. 357-360.

5. Duchen, M.R., et al., On the involvement of a cyclosporin A sensitive mitochondrial pore in myocardial reperfusion injury. Cardiovasc Res, 1993. 27(10): p. 1790-4.

6. Griffiths, E.J. and A.P. Halestrap, Mitochondrial non-specific pores remain closed during cardiac ischaemia, but open upon reperfusion. The Biochemical journal, 1995. 307 ( Pt 1): p. 93-98.

7. Irwin, W.A., et al., Mitochondrial dysfunction and apoptosis in myopathic mice with collagen VI deficiency. Nature genetics, 2003. 35: p. 367-371.

8. Argaud, L., et al., Specific inhibition of the mitochondrial permeability transition prevents lethal reperfusion injury. Journal of molecular and cellular cardiology, 2005. 38: p. 367-374.

9. $\mathrm{Du}, \mathrm{H}$., et al., Cyclophilin D deficiency attenuates mitochondrial and neuronal perturbation and ameliorates learning and memory in Alzheimer's disease. Nat Med, 2008. 14(10): p. 1097-105.

10. Okada, M., et al., Long-term effects of edaravone on survival of patients with amyotrophic lateral sclerosis. eNeurologicalSci, 2018. 11: p. 11-14.

11. Merlini, L., et al., Cyclosporin A corrects mitochondrial dysfunction and muscle apoptosis in patients with collagen VI myopathies. Proceedings of the National Academy of Sciences of the United States of America, 2008. 105: p. 5225-5229.

12. Roy, S., et al., Discovery, Synthesis, and Optimization of Diarylisoxazole-3-carboxamides as Potent Inhibitors of the Mitochondrial Permeability Transition Pore. ChemMedChem, 2015. 10: p. 1655-1671.

13. Schiavone, M., et al., Alisporivir rescues defective mitochondrial respiration in Duchenne muscular dystrophy. Pharmacol Res, 2017. 125(Pt B): p. 122-131.

14. Baines, C.P. and M. Gutiérrez-Aguilar, The still uncertain identity of the channel-forming unit(s) of the mitochondrial permeability transition pore. Cell Calcium, 2018. 73: p. 121-130.

15. Crompton, M., S. Virji, and J.M. Ward, Cyclophilin-D binds strongly to complexes of the voltage-dependent anion channel and the adenine nucleotide translocase to form the permeability transition pore. Eur J Biochem, 1998. 258(2): p. 729-35.

16. Leung, A.W., P. Varanyuwatana, and A.P. Halestrap, The mitochondrial phosphate carrier interacts with cyclophilin D and may play a key role in the permeability transition. J Biol Chem, 2008. 283(39): p. 26312-23. 
17. Shanmughapriya, S., et al., SPG7 Is an Essential and Conserved Component of the Mitochondrial Permeability Transition Pore. Mol Cell, 2015. 60(1): p. 47-62.

18. Giorgio, V., et al., Dimers of mitochondrial ATP synthase form the permeability transition pore. Proceedings of the National Academy of Sciences of the United States of America, 2013. 110: p. 5887-5892.

19. Alavian, K.N., et al., An uncoupling channel within the c-subunit ring of the F1FO ATP synthase is the mitochondrial permeability transition pore. Proceedings of the National Academy of Sciences of the United States of America, 2014. 111: p. 10580-10585.

20. Karch, J., et al., Bax and Bak function as the outer membrane component of the mitochondrial permeability pore in regulating necrotic cell death in mice. Elife, 2013. 2: p. e00772.

21. Baines, C.P., et al., Voltage-dependent anion channels are dispensable for mitochondrialdependent cell death. Nature Cell Biology, 2007. 9: p. 550.

22. He, J., et al., Permeability transition in human mitochondria persists in the absence of peripheral stalk subunits of ATP synthase. Proceedings of the National Academy of Sciences, 2017. 114(34): p. 9086-9091.

23. He, J., et al., Persistence of the mitochondrial permeability transition in the absence of subunit c of human ATP synthase. Proceedings of the National Academy of Sciences, 2017. 114(13): p. 3409-3414.

24. Bernardi, P. and M. Forte, Commentary: SPG7 is an essential and conserved component of the mitochondrial permeability transition pore. Frontiers in Physiology, 2015. 6(320).

25. Kokoszka, J.E., et al., The ADP/ATP translocator is not essential for the mitochondrial permeability transition pore. Nature, 2004. 427(6973): p. 461-5.

26. Kwong, J.Q., et al., Genetic deletion of the mitochondrial phosphate carrier desensitizes the mitochondrial permeability transition pore and causes cardiomyopathy. Cell Death Differ, 2014. 21(8): p. 1209-17.

27. Baines, C.P., et al., Loss of cyclophilin D reveals a critical role for mitochondrial permeability transition in cell death. Nature, 2005. 434: p. 658-662.

28. Nakagawa, T., et al., Cyclophilin D-dependent mitochondrial permeability transition regulates some necrotic but not apoptotic cell death. Nature, 2005. 434(7033): p. 652-8.

29. Schinzel, A.C., et al., Cyclophilin D is a component of mitochondrial permeability transition and mediates neuronal cell death after focal cerebral ischemia. Proceedings of the National Academy of Sciences of the United States of America, 2005. 102(34): p. 12005-12010.

30. Forte, M., et al., Cyclophilin D inactivation protects axons in experimental autoimmune encephalomyelitis, an animal model of multiple sclerosis. Proceedings of the National Academy of Sciences, 2007. 104(18): p. 7558-7563.

31. Millay, D.P., et al., Genetic and pharmacologic inhibition of mitochondrial-dependent necrosis attenuates muscular dystrophy. Nat Med, 2008. 14(4): p. 442-7.

32. Devalaraja-Narashimha, K., A.M. Diener, and B.J. Padanilam, Cyclophilin D gene ablation protects mice from ischemic renal injury. Am J Physiol Renal Physiol, 2009. 297(3): p. F74959.

33. Palma, E., et al., Genetic ablation of cyclophilin D rescues mitochondrial defects and prevents muscle apoptosis in collagen VI myopathic mice. Hum Mol Genet, 2009. 18(11): p. 2024-31.

34. Du, H., et al., Cyclophilin D deficiency improves mitochondrial function and learning/memory in aging Alzheimer disease mouse model. Neurobiol Aging, 2011. 32(3): p. 398-406.

35. Carraro, M., et al., Channel formation by yeast F-ATP synthase and the role of dimerization in the mitochondrial permeability transition. The Journal of biological chemistry, 2014. 289: $p$. 15980-15985.

36. Giorgio, V., et al., $\mathrm{Ca}(2+)$ binding to F-ATP synthase beta subunit triggers the mitochondrial permeability transition. EMBO Rep, 2017. 18(7): p. 1065-1076. 
37. Antoniel, M., et al., The unique histidine in OSCP subunit of F-ATP synthase mediates inhibition of the permeability transition pore by acidic pH. EMBO Rep, 2018. 19(2): p. 257268.

38. Zhou, W., et al., Atomistic simulations indicate the c-subunit ring of the F1Fo ATP synthase is not the mitochondrial permeability transition pore. Elife, 2017. 6.

39. Chinopoulos, C., Mitochondrial permeability transition pore: Back to the drawing board. Neurochemistry International, 2018. 117: p. 49-54.

40. Luvisetto, S., et al., Enhancement of anxiety, facilitation of avoidance behavior, and occurrence of adult-onset obesity in mice lacking mitochondrial cyclophilin D. Neuroscience, 2008. 155(3): p. 585-96.

41. LoGuidice, A., et al., Pharmacologic targeting or genetic deletion of mitochondrial cyclophilin $D$ protects from NSAID-induced small intestinal ulceration in mice. Toxicol Sci, 2010. 118(1): p. 276-85.

42. Shum, L.C., et al., Cyclophilin D Knock-Out Mice Show Enhanced Resistance to Osteoporosis and to Metabolic Changes Observed in Aging Bone. PLoS One, 2016. 11(5): p. e0155709.

43. Martin, L.J., et al., The mitochondrial permeability transition pore in motor neurons: involvement in the pathobiology of ALS mice. Exp Neurol, 2009. 218(2): p. 333-46.

44. Berman, S.B., S.C. Watkins, and T.G. Hastings, Quantitative biochemical and ultrastructural comparison of mitochondrial permeability transition in isolated brain and liver mitochondria: evidence for reduced sensitivity of brain mitochondria. Exp Neurol, 2000. 164(2): p. 415-25.

45. Endlicher, R., et al., Tissue specific sensitivity of mitochondrial permeability transition pore to Ca2+ ions. Acta Medica (Hradec Kralove), 2009. 52(2): p. 69-72.

46. Azzolin, L., et al., The mitochondrial permeability transition from yeast to mammals. FEBS Lett, 2010. 584(12): p. 2504-9.

47. Uribe-Carvajal, S., et al., Mitochondrial Unselective Channels throughout the eukaryotic domain. Mitochondrion, 2011. 11(3): p. 382-90.

48. Vianello, A., et al., The mitochondrial permeability transition pore (PTP) - an example of multiple molecular exaptation? Biochim Biophys Acta, 2012. 1817(11): p. 2072-86.

49. Ayoub, I.M., J. Radhakrishnan, and R.J. Gazmuri, In vivo opening of the mitochondrial permeability transition pore in a rat model of ventricular fibrillation and closed-chest resuscitation. Am J Transl Res, 2017. 9(7): p. 3345-3359.

50. Kerr, P.M., M.S. Suleiman, and A.P. Halestrap, Reversal of permeability transition during recovery of hearts from ischemia and its enhancement by pyruvate. Am J Physiol, 1999. 276(2 Pt 2): p. H496-502.

51. Hansson, M.J., et al., Powerful cyclosporin inhibition of calcium-induced permeability transition in brain mitochondria. Brain Res, 2003. 960(1-2): p. 99-111.

52. Hazelton, J.L., et al., Cyclophilin D is expressed predominantly in mitochondria of gammaaminobutyric acidergic interneurons. J Neurosci Res, 2009. 87(5): p. 1250-9.

53. Lam, C.K., et al., HAX-1 regulates cyclophilin-D levels and mitochondria permeability transition pore in the heart. Proc Natl Acad Sci U S A, 2015. 112(47): p. E6466-75.

54. Kang, B.H., et al., Regulation of Tumor Cell Mitochondrial Homeostasis by an OrganelleSpecific Hsp90 Chaperone Network. Cell, 2007. 131(2): p. 257-270.

55. Davis, T.L., et al., Structural and Biochemical Characterization of the Human Cyclophilin Family of Peptidyl-Prolyl Isomerases. PLOS Biology, 2010. 8(7): p. e1000439.

56. Gutiérrez-Aguilar, M. and C.P. Baines, Structural mechanisms of cyclophilin D-dependent control of the mitochondrial permeability transition pore. Biochimica et Biophysica Acta (BBA) - General Subjects, 2015. 1850(10): p. 2041-2047.

57. Taylor, P., et al., Structures of cyclophilin-ligand complexes. Prog Biophys Mol Biol, 1997. 67(2-3): p. 155-81.

58. Barbarino, J.M., et al., PharmGKB summary: cyclosporine and tacrolimus pathways. Pharmacogenetics and genomics, 2013. 23(10): p. 563-585. 
59. Griffiths, E.J. and A.P. Halestrap, Protection by Cyclosporin A of Ischemia/ReperfusionInduced Damage in Isolated Rat Hearts. Journal of Molecular and Cellular Cardiology, 1993. 25(12): p. 1461-1469.

60. Nazareth, W., N. Yafei, and M. Crompton, Inhibition of anoxia-induced injury in heart myocytes by cyclosporin A. Journal of Molecular and Cellular Cardiology, 1991. 23(12): p. 1351-1354.

61. Waldmeier, P.C., et al., Inhibition of the mitochondrial permeability transition by the nonimmunosuppressive cyclosporin derivative NIM811. Mol Pharmacol, 2002. 62(1): p. 22-9.

62. Hansson, M.J., et al., The nonimmunosuppressive cyclosporin analogs NIM811 and UNILO25 display nanomolar potencies on permeability transition in brain-derived mitochondria. Journal of bioenergetics and biomembranes, 2004. 36: p. 407-413.

63. Zulian, A., et al., NIM811, a cyclophilin inhibitor without immunosuppressive activity, is beneficial in collagen VI congenital muscular dystrophy models. Hum Mol Genet, 2014. 23(20): p. 5353-63.

64. Tiepolo, T., et al., The cyclophilin inhibitor Debio 025 normalizes mitochondrial function, muscle apoptosis and ultrastructural defects in Col6a1-/- myopathic mice. British journal of pharmacology, 2009. 157: p. 1045-1052.

65. Reutenauer, J., et al., Investigation of Debio 025, a cyclophilin inhibitor, in the dystrophic mdx mouse, a model for Duchenne muscular dystrophy. Br J Pharmacol, 2008. 155(4): p. 574-84.

66. Wissing, E.R., et al., Debio-025 is more effective than prednisone in reducing muscular pathology in mdx mice. Neuromuscul Disord, 2010. 20(11): p. 753-60.

67. Clarke, S.J., G.P. McStay, and A.P. Halestrap, Sanglifehrin A acts as a potent inhibitor of the mitochondrial permeability transition and reperfusion injury of the heart by binding to cyclophilin-D at a different site from cyclosporin A. The Journal of biological chemistry, 2002. 277: p. 34793-34799.

68. Shore, E.R., et al., Small Molecule Inhibitors of Cyclophilin D To Protect Mitochondrial Function as a Potential Treatment for Acute Pancreatitis. J Med Chem, 2016. 59(6): p. 2596611.

69. Park, I., et al., Discovery of non-peptidic small molecule inhibitors of cyclophilin D as neuroprotective agents in Abeta-induced mitochondrial dysfunction. J Comput Aided Mol Des, 2017. 31(10): p. 929-941.

70. Valasani, K.R., et al., Structure based design, synthesis, pharmacophore modeling, virtual screening, and molecular docking studies for identification of novel cyclophilin D inhibitors. J Chem Inf Model, 2014. 54(3): p. 902-12.

71. Valasani, K.R., et al., Identification of a Small Molecule Cyclophilin D Inhibitor for Rescuing Abeta-Mediated Mitochondrial Dysfunction. ACS Med Chem Lett, 2016. 7(3): p. 294-9.

72. Xie, L., et al., The novel cyclophilin D inhibitor compound 19 protects retinal pigment epithelium cells and retinal ganglion cells from UV radiation. Biochem Biophys Res Commun, 2017. 487(4): p. 807-812.

73. Schaller, S., et al., TRO40303, a new cardioprotective compound, inhibits mitochondrial permeability transition. The Journal of pharmacology and experimental therapeutics, 2010. 333: p. 696-706.

74. Šileikytè, J., et al., Regulation of the Mitochondrial Permeability Transition Pore by the Outer Membrane Does Not Involve the Peripheral Benzodiazepine Receptor (Translocator Protein of 18 kDa (TSPO)). Journal of Biological Chemistry, 2014. 289(20): p. 13769-13781.

75. Fancelli, D., et al., Cinnamic anilides as new mitochondrial permeability transition pore inhibitors endowed with ischemia-reperfusion injury protective effect in vivo. Journal of medicinal chemistry, 2014. 57: p. 5333-5347.

76. Richardson, A.P. and A.P. Halestrap, Quantification of active mitochondrial permeability transition pores using GNX-4975 inhibitor titrations provides insights into molecular identity. Biochem J, 2016. 473(9): p. 1129-40. 
77. Halestrap, A.P. and A.P. Richardson, The mitochondrial permeability transition: a current perspective on its identity and role in ischaemia/reperfusion injury. J Mol Cell Cardiol, 2015. 78: p. 129-41.

78. Martin, L.J., et al., GNX-4728, a novel small molecule drug inhibitor of mitochondrial permeability transition, is therapeutic in a mouse model of amyotrophic lateral sclerosis. Frontiers in cellular neuroscience, 2014. 8: p. 433.

79. Sileikyte, J., et al., Small Molecules Targeting the Mitochondrial Permeability Transition, in Probe Reports from the NIH Molecular Libraries Program. 2010, National Center for Biotechnology Information (US): Bethesda (MD).

80. Roy, S., et al., N-Phenylbenzamides as Potent Inhibitors of the Mitochondrial Permeability Transition Pore. ChemMedChem, 2016. 11: p. 283-288.

81. Briston, T., et al., Identification of ER-000444793, a Cyclophilin D-independent inhibitor of mitochondrial permeability transition, using a high-throughput screen in cryopreserved mitochondria. Sci Rep, 2016. 6: p. 37798.

82. Teixeira, J., et al., Discovery of a new mitochondria permeability transition pore (MPTP) inhibitor based on gallic acid. J Enzyme Inhib Med Chem, 2018. 33(1): p. 567-576.

83. Hwang, I.-C., et al., Therapeutic Potential of a Novel Necrosis Inhibitor, 7-Amino-Indole, in Myocardial Ischemia-Reperfusion Injury. Hypertension, 2018. 71(6): p. 1143-1155.

84. Kon, N., A. Satoh, and N. Miyoshi, A small-molecule DS44170716 inhibits Ca(2+)-induced mitochondrial permeability transition. Scientific Reports, 2017. 7: p. 3864.

85. Rajesh, K.G., et al., Antioxidant MCl-186 inhibits mitochondrial permeability transition pore and upregulates Bcl-2 expression. Am J Physiol Heart Circ Physiol, 2003. 285(5): p. H2171-8.

86. Takayasu, Y., et al., Edaravone, a radical scavenger, inhibits mitochondrial permeability transition pore in rat brain. J Pharmacol Sci, 2007. 103(4): p. 434-7.

87. Cesura, A.M., et al., The voltage-dependent anion channel is the target for a new class of inhibitors of the mitochondrial permeability transition pore. J Biol Chem, 2003. 278(50): $p$. 49812-8.

88. Krauskopf, A., et al., Properties of the permeability transition in VDAC1-/- mitochondria. Biochimica et Biophysica Acta (BBA) - Bioenergetics, 2006. 1757(5): p. 590-595.

89. Zhang, Chung, and Oldenburg, A Simple Statistical Parameter for Use in Evaluation and Validation of High Throughput Screening Assays. Journal of biomolecular screening, 1999. 4: p. 67-73.

90. Moffat, J.G., et al., Opportunities and challenges in phenotypic drug discovery: an industry perspective. Nature Reviews Drug Discovery, 2017. 16: p. 531.

91. Cung, T.-T., et al., Cyclosporine before $\mathrm{PCl}$ in Patients with Acute Myocardial Infarction. The New England journal of medicine, 2015. 373: p. 1021-1031.

92. Kwong, Jennifer Q. and Jeffery D. Molkentin, Physiological and Pathological Roles of the Mitochondrial Permeability Transition Pore in the Heart. Cell Metabolism, 2015. 21(2): p. 206-214.

93. Altschuld, R.A., et al., Cyclosporin inhibits mitochondrial calcium efflux in isolated adult rat ventricular cardiomyocytes. Am J Physiol, 1992. 262 (6 Pt 2): p. H1699-704.

94. Hom, Jennifer R., et al., The Permeability Transition Pore Controls Cardiac Mitochondrial Maturation and Myocyte Differentiation. Developmental Cell, 2011. 21(3): p. 469-478.

95. Ko, Y.H., et al., Mitochondrial ATP synthasome: Cristae-enriched membranes and a multiwell detergent screening assay yield dispersed single complexes containing the ATP synthase and carriers for Pi and ADP/ATP. Journal of Biological Chemistry, 2003. 278(14): p. 12305-12309.

96. Giorgio, V., et al., Cyclophilin D modulates mitochondrial FOF1-ATP synthase by interacting with the lateral stalk of the complex. The Journal of biological chemistry, 2009. 284: $p$. 33982-33988.

97. Beutner, G., R.E. Alanzalon, and G.A. Porter, Cyclophilin D regulates the dynamic assembly of mitochondrial ATP synthase into synthasomes. Scientific Reports, 2017. 7(1): p. 14488. 
98. Duchen, M.R., A. Verkhratsky, and S. Muallem, Mitochondria and calcium in health and disease. Cell calcium, 2008. 44: p. 1-5.

99. De Marchi, E., et al., The mitochondrial permeability transition pore is a dispensable element for mitochondrial calcium efflux. Cell Calcium, 2014. 56(1): p. 1-13.

100. Elrod, J.W., et al., Cyclophilin D controls mitochondrial pore-dependent Ca2+exchange, metabolic flexibility, and propensity for heart failure in mice. The Journal of Clinical Investigation, 2010. 120(10): p. 3680-3687.

101. Malouitre, S., et al., Mitochondrial targeting of cyclosporin A enables selective inhibition of cyclophilin-D and enhanced cytoprotection after glucose and oxygen deprivation. Biochemical Journal, 2010. 425(1): p. 137.

102. Jin, L. and S.C. Harrison, Crystal structure of human calcineurin complexed with cyclosporin A and human cyclophilin. Proceedings of the National Academy of Sciences, 2002. 99(21): p. 13522.

103. Warne, J., et al., Selective inhibition of the mitochondrial permeability transition pore protects against neuro-degeneration in experimental multiple sclerosis. The Journal of biological chemistry, 2015.

104. Sakamoto, K.M., et al., Protacs: Chimeric molecules that target proteins to the Skp1-Cullin-F box complex for ubiquitination and degradation. Proceedings of the National Academy of Sciences, 2001. 98(15): p. 8554.

105. Lebraud, H., et al., Protein Degradation by In-Cell Self-Assembly of Proteolysis Targeting Chimeras. ACS Central Science, 2016. 2(12): p. 927-934.

106. Crooke, S.T., et al., RNA-Targeted Therapeutics. Cell Metabolism, 2018. 27(4): p. 714-739.

107. Connern, C.P. and A.P. Halestrap, Purification and N-terminal sequencing of peptidyl-prolyl cis-trans-isomerase from rat liver mitochondrial matrix reveals the existence of a distinct mitochondrial cyclophilin. The Biochemical journal, 1992. 284 (Pt 2): p. 381-385.

108. Woodfield, K., et al., Direct demonstration of a specific interaction between cyclophilin-D and the adenine nucleotide translocase confirms their role in the mitochondrial permeability transition. Biochemical Journal, 1998. 336(2): p. 287-290.

109. Vaseva, Angelina V., et al., p53 Opens the Mitochondrial Permeability Transition Pore to Trigger Necrosis. Cell, 2012. 149(7): p. 1536-1548.

110. McGee, Allison M. and Christopher P. Baines, Complement 1q-binding protein inhibits the mitochondrial permeability transition pore and protects against oxidative stress-induced death. Biochemical Journal, 2011. 433(1): p. 119.

111. Paillard, M., et al., Depressing mitochondria-reticulum interactions protects cardiomyocytes from lethal hypoxia-reoxygenation injury. Circulation, 2013. 128(14): p. 1555-65.

112. Lee, C.F., et al., Normalization of NAD+ Redox Balance as a Therapy for Heart Failure. Circulation, 2016. 134(12): p. 883-94.

113. Nguyen, T.T., et al., Cysteine 203 of Cyclophilin D Is Critical for Cyclophilin D Activation of the Mitochondrial Permeability Transition Pore. Journal of Biological Chemistry, 2011. 286(46): p. 40184-40192.

114. Rasola, A., et al., Activation of mitochondrial ERK protects cancer cells from death through inhibition of the permeability transition. Proceedings of the National Academy of Sciences, 2010. 107(2): p. 726.

115. Hafner, A.V., et al., Regulation of the MPTP by SIRT3-mediated deacetylation of CypD at lysine 166 suppresses age-related cardiac hypertrophy. Aging, 2010. 2(12): p. 914-923.

116. Parks, R.J., et al., Cyclophilin D-mediated regulation of the permeability transition pore is altered in mice lacking the mitochondrial calcium uniporter. Cardiovasc Res, 2018.

117. Piot, C., et al., Effect of cyclosporine on reperfusion injury in acute myocardial infarction. $\mathrm{N}$ Engl J Med, 2008. 359(5): p. 473-81.

118. Mewton, N., et al., Effect of Cyclosporine on Left Ventricular Remodeling After Reperfused Myocardial Infarction. Journal of the American College of Cardiology, 2010. 55(12): p. 1200. 
119. Chiari, P., et al., Cyclosporine protects the heart during aortic valve surgery. Anesthesiology, 2014. 121(2): p. 232-8.

120. Hausenloy, D., et al., The effect of cyclosporin-A on peri-operative myocardial injury in adult patients undergoing coronary artery bypass graft surgery: a randomised controlled clinical trial. Heart, 2014. 100(7): p. 544-549.

121. Ghaffari, S., et al., The Effect of Prethrombolytic Cyclosporine-A Injection on Clinical Outcome of Acute Anterior ST-Elevation Myocardial Infarction. Cardiovascular Therapeutics, 2012. 31(4): p. e34-e39.

122. Ottani, F., et al., Cyclosporine A in Reperfused Myocardial Infarction. Journal of the American College of Cardiology, 2016. 67(4): p. 365.

123. Bernardi, P. and F. Di Lisa, Cyclosporine before PCl in Acute Myocardial Infarction. N Engl J Med, 2016. 374(1): p. 89-90.

124. Ederoth, P., et al., Cyclosporine before Coronary Artery Bypass Grafting Does Not Prevent Postoperative Decreases in Renal FunctionA Randomized Clinical Trial. Anesthesiology, 2018. 128(4): p. 710-717.

125. Atar, D., et al., Effect of intravenous TRO40303 as an adjunct to primary percutaneous coronary intervention for acute ST-elevation myocardial infarction: MITOCARE study results. Eur Heart J, 2015. 36(2): p. 112-9.

126. Butt, N., et al., Administration of the Mitochondrial Permeability Transition Pore Inhibitor, TRO40303, prior to Primary Percutaneous Coronary Intervention, Does Not Affect the Levels of Pro-Inflammatory Cytokines or Acute-Phase Proteins. Cardiology, 2017. 138(2): p. 122132.

127. Trankle, C., et al., Mitochondrial Membrane Permeability Inhibitors in Acute Myocardial Infarction. JACC: Basic to Translational Science, 2016. 1(6): p. 524.

128. Monassier, L., et al., Targeting myocardial reperfusion injuries with cyclosporine in the CIRCUS Trial - pharmacological reasons for failure. Fundam Clin Pharmacol, 2016. 30(2): p. 191-3.

129. Abe K, A.M., Tsuji S, Itoyama Y, Sobue G, Togo M, Hamada C, Tanaka M, Akimoto M, Nakamura K, Takahashi F, Kondo K, Yoshino H, Abe K, Aoki M, Tsuji S, Itoyama Y, Sobue G, Togo M, Hamada C, Sasaki H, Yabe I, Doi S, Warita H, Imai T, Ito H, Fukuchi M, Osumi E, Wada M, Nakano I, Morita M, Ogata K, Maruki Y, Ito K, Kano O, Yamazaki M, Takahashi Y, Ishiura H, Ogino M, Koike R, Ishida C, Uchiyama T, Mizoguchi K, Obi T, Watanabe H, Atsuta N, Aiba I, Taniguchi A, Sawada H, Hazama T, Fujimura H, Kusaka H, Kunieda T, Kikuchi H, Matsuo H, Ueyama H, Uekawa K, Tanaka M, Akimoto M, Ueda M, Murakami A, Sumii R, Kudou T, Nakamura K, Morimoto K, Yoneoka T, Hirai M, Sasaki K, Terai H, Natori T, Matsui H, Kotani K, Yoshida K, Iwasaki T, Takahashi F, Kondo K, Yoshino H., Safety and efficacy of edaravone in well defined patients with amyotrophic lateral sclerosis: a randomised, doubleblind, placebo-controlled trial. Lancet Neurol, 2017. 16(7): p. 505-512.

130. Angelin, A., P. Bonaldo, and P. Bernardi, Altered threshold of the mitochondrial permeability transition pore in Ullrich congenital muscular dystrophy. Biochimica et biophysica acta, 2008. 1777: p. 893-896.

131. Brand, M.D. and D.G. Nicholls, Assessing mitochondrial dysfunction in cells. The Biochemical journal, 2011. 435: p. 297-312.

132. Duchen, M.R., A. Surin, and J. Jacobson, Imaging mitochondrial function in intact cells, in Methods in Enzymology. 2003, Academic Press. p. 353-389.

133. Traba, J., et al., SCaMC-1 promotes cancer cell survival by desensitizing mitochondrial permeability transition via ATP/ADP-mediated matrix $\mathrm{Ca}(2+)$ buffering. Cell Death Differ, 2012. 19(4): p. 650-60.

134. Bernardi, P., et al., Modulation of the mitochondrial permeability transition pore. Effect of protons and divalent cations. The Journal of biological chemistry, 1992. 267: p. 2934-2939. 
135. Keep, M., et al., Intrathecal cyclosporin prolongs survival of late-stage ALS mice. Brain Res, 2001. 894(2): p. 327-31.

136. Gauba, E., L. Guo, and H. Du, Cyclophilin D Promotes Brain Mitochondrial F1FO ATP Synthase Dysfunction in Aging Mice. J Alzheimers Dis, 2017. 55(4): p. 1351-1362.

137. Pillot, B., et al., NPV019 potently inhibits cyclophilin D-dependent mitochondrial permeability transition in human heart and reduces myocardial infarct size in mice. Journal of the American College of Cardiology, 2015. 65(10 Supplement): p. A159.

138. Morciano, G., et al., Discovery of novel 1,3,8-triazaspiro[4.5]decane derivatives that target the $c$ subunit of F1/FO-adenosine triphosphate (ATP) synthase for the treatment of reperfusion damage in myocardial infarction. J Med Chem, 2018.

139. Kon, N., et al., DS16570511 is a small-molecule inhibitor of the mitochondrial calcium uniporter. Cell Death Discov, 2017. 3: p. 17045.

\section{Figure Legends}

Figure 1; Key Figure: Physiological and pharmacological regulation of the mPTP complex.

The MPTP is a proteinaceous complex, of unresolved bio-architecture. Although high matrix $\mathrm{Ca}^{2+}$ is the principal triggering event for mPTP opening, a number of physiological factors and pharmacological agents can influence probability of pore opening. In addition to extremes of $\mathrm{Ca}^{2+}, \mathrm{mPTP}$ opening is further shaped by the cellular environment; low $\mathrm{pH}$, adenine nucleotides and $\mathrm{Mg}^{2+}$ negatively regulate pore opening and conversely $\mathrm{Ca}^{2+}$ overload, adenine nucleotide depletion and reactive oxygen species (ROS) promote channel opening $[6,134,135]$. Small molecule inhibitors have been described as having both CypD-dependent and -independent mechanisms. Abbreviations: A $\beta$; amyloid beta, C1QBP; complement C1q binding protein, ETC; electron transport chain, $\mathrm{MCU}$; mitochondrial $\mathrm{Ca}^{2+}$ uniporter

(Box 2) Figure I: Measurement of mitochondrial transmembrane potential using TMRM. (A) At low concentration, transmembrane potential depolarisation results in TMRM redistribution. (B) At high concentration, transmembrane potential depolarisation results in relief of dye quenching and increased fluorescence. To ensure correct interpretation and meaningful data, careful consideration of experimental hypothesis and bioassay conditions is necessary to ensure correct use of membrane potential dyes.

Table 1: Phenotype of Ppif-- in disease models 


\begin{tabular}{|c|c|c|}
\hline Disease model & Phenotype of Ppif $^{/-}$ & Reference \\
\hline Aging & $\begin{array}{c}\text { (1) } \downarrow F_{1} F_{0} \text {-ATP synthase enzymatic decline (2) } \downarrow \\
\text { F }_{1} F_{0}-A T P \text { synthase uncoupling (3) maintained } \\
\text { respiratory control ratio (RCR) }\end{array}$ & [136] \\
\hline Aging; osteoporosis & $\begin{array}{c}\text { (1) normal/rescued trabecular bone volume and } \\
\text { cortical thickness ( } 2 \text { ) resistance against age-related } \\
\text { bone loss }\end{array}$ & [42] \\
\hline $\begin{array}{l}\text { Amyotrophic lateral sclerosis; } \\
\text { mSOD1-G93A }\end{array}$ & (1) $\uparrow$ survival (2) delayed onset of disease & [43] \\
\hline $\begin{array}{l}\text { Alzheimer's disease; mutant } \\
\text { amyloid precursor protein } \\
\text { (APP) }\end{array}$ & (1) $\uparrow$ spatial learning and memory & {$[34]$} \\
\hline $\begin{array}{l}\text { Enteropathy; diclofenac- } \\
\text { induced }\end{array}$ & $\begin{array}{c}\text { (1) } \downarrow \text { mucosal injury; (2) normal/rescued serum } \\
\text { alkaline phosphatase }\end{array}$ & [41] \\
\hline $\begin{array}{c}\text { Multiple sclerosis; } \\
\text { experimental allergic } \\
\text { encephalomyelitis (EAE) }\end{array}$ & $\begin{array}{c}\text { (1) Clinical improvement [ } \downarrow \text { EAE score]; (2) axonal } \\
\text { preservation }\end{array}$ & [30] \\
\hline $\begin{array}{l}\text { Muscular dystrophy; collagen } \\
\qquad \text { VI deficiency }\end{array}$ & $\begin{array}{l}\text { (1) } \downarrow \text { myofibre degeneration; (2) } \uparrow \text { mitochondrial } \\
\text { function; (3) normalized apoptosis; (4) } \downarrow \\
\text { permeability of skeletal muscle fibres }\end{array}$ & [33] \\
\hline $\begin{array}{l}\text { Muscular dystrophy; laminin- } \\
\qquad 2 \text { deficiency }\end{array}$ & $\begin{array}{c}\text { (1) } \text { survival; (2) } \uparrow \text { life span; (3) } \uparrow \text { total fibre } \\
\text { number; (4) } \uparrow \text { body weight }\end{array}$ & [31] \\
\hline $\begin{array}{l}\text { Muscular dystrophy; } \\
\text { sarcoglycan deficiency }\end{array}$ & $\begin{array}{c}\text { (1) } \downarrow \text { myofibre degeneration (2); } \downarrow \text { cardiac fibrosis; } \\
\text { (3) normalised muscle mass; (4) } \downarrow \text { inflammation }\end{array}$ & [31] \\
\hline $\begin{array}{l}\text { Myocardial infarction: } \\
\text { ischaemia-reperfusion injury }\end{array}$ & (1) $\downarrow$ infarct size; (2) $\downarrow$ LDH release & {$[27,28]$} \\
\hline Renal ischaemia & $\begin{array}{c}\text { (1) } \downarrow \text { tubular cell necrosis; (2) } \downarrow \text { tubular dilatation; } \\
\text { (3) } \downarrow \text { erythrocyte congestion }\end{array}$ & [32] \\
\hline $\begin{array}{l}\text { Stroke: acute middle cerebral } \\
\text { artery occlusion and } \\
\text { reperfusion }\end{array}$ & (1) neuroprotection; (2) $\downarrow$ infarct size & [29] \\
\hline
\end{tabular}

Table 2: mPTP inhibitors, including macrocyclic CypD inhibitors, small molecule CypD inhibitors and small molecule inhibitors of the MPTP working by non-CypD mechanisms. ND: not disclosed, ETC: electron transport chain. 


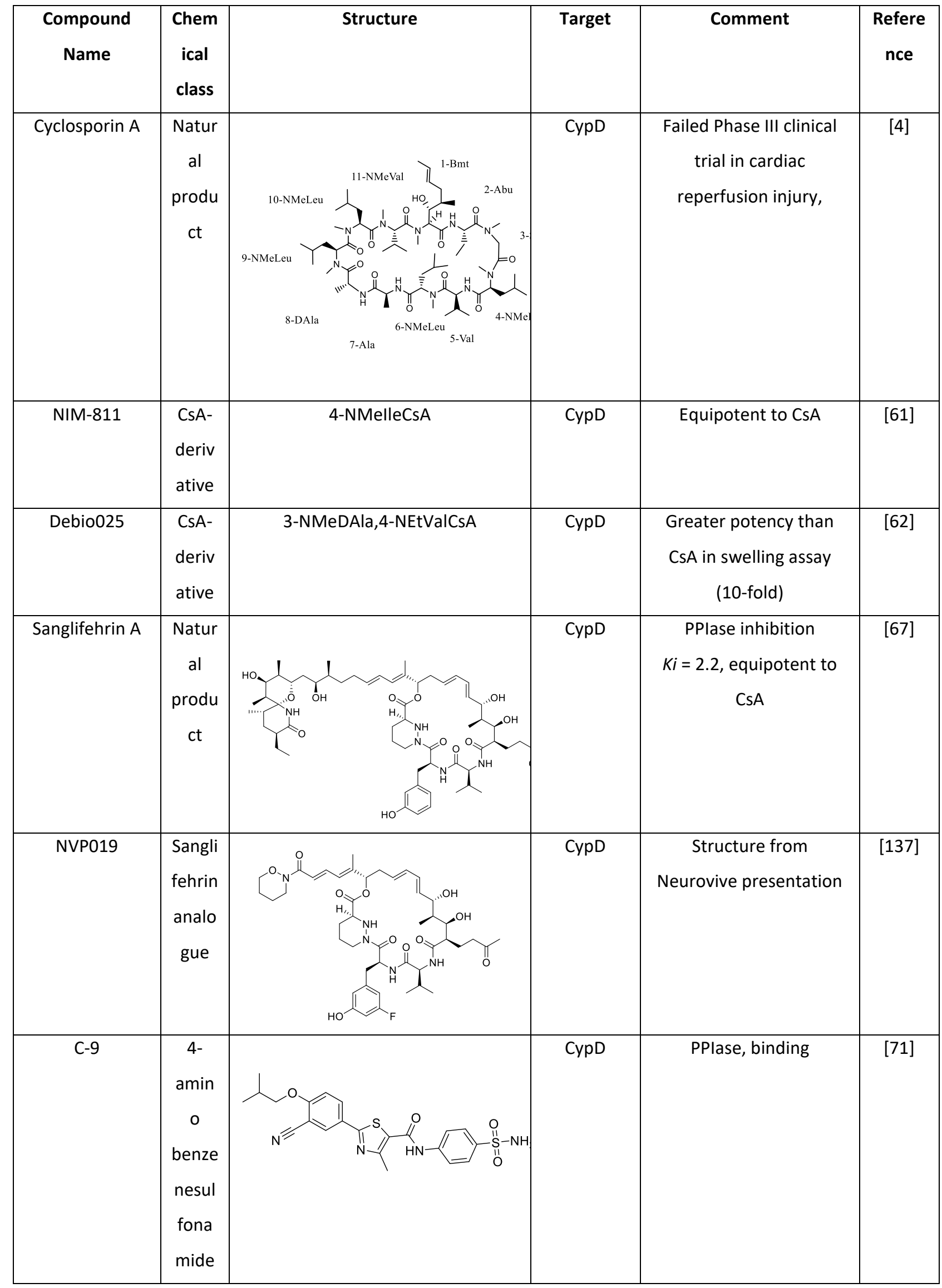




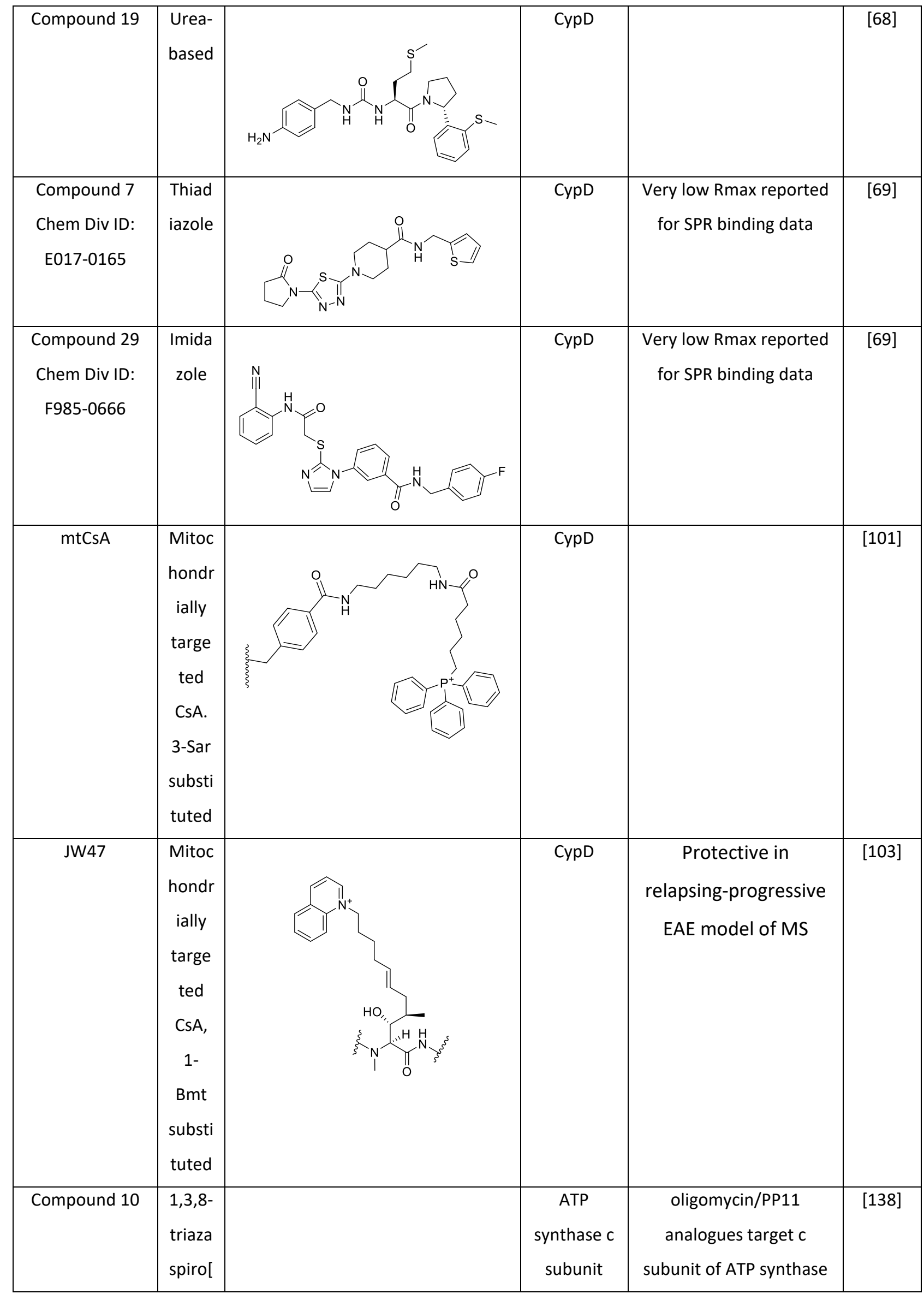




\begin{tabular}{|c|c|c|c|c|c|}
\hline & $\begin{array}{l}4.5] \mathrm{d} \\
\text { ecane }\end{array}$ & (N) & & & \\
\hline TRO-40303 & $\begin{array}{l}\text { Steroi } \\
\mathrm{d} \\
\text { analo } \\
\text { gue }\end{array}$ & & TSPO(+) & $\begin{array}{c}\text { Failed to limit } \\
\text { myocardial injury } \\
\text { following reperfusion } \\
\text { in Phase Il study }\end{array}$ & [73] \\
\hline Compound 4 & $\begin{array}{c}N- \\
\text { phen } \\
y l- \\
\text { benza } \\
\text { mide }\end{array}$ & & ND & & {$[80]$} \\
\hline Compound 22 & $\begin{array}{l}\text { Cinna } \\
\text { mic } \\
\text { anilid } \\
\mathrm{e}\end{array}$ & & ND & & [75] \\
\hline GNX-4975 & $\begin{array}{c}\text { Cinna } \\
\text { mic } \\
\text { anilid } \\
\text { e }\end{array}$ & $\begin{array}{l}\text { Structure unclear from publication } \\
\text { but belongs to same class as } \\
\text { compound } 22 \text { above (maybe } \\
\text { identical). }\end{array}$ & $\begin{array}{l}\text { Stabilisatio } \\
\mathrm{n} \text { of PiC } \\
\text { and c-ANT } \\
\text { interaction }\end{array}$ & $\begin{array}{c}2 \text { fold increase in } \\
\text { lifespan G37R-human } \\
\text { mSOD-1 mouse }\end{array}$ & $\begin{array}{l}76, \\
78]\end{array}$ \\
\hline $\begin{array}{l}\mathrm{N} \text {-(3-chloro-2- } \\
\text { methylphenyl)- } \\
\text { 5-(4-fluoro-3- } \\
\text { hydroxyphenyl) } \\
\text { isoxazole-3- } \\
\text { carboxamide }\end{array}$ & $\begin{array}{l}\text { Isoxa } \\
\text { zole }\end{array}$ & & ND & $\begin{array}{c}\text { Improved muscle } \\
\text { function and structure } \\
\text { in mutant collagen VI } \\
\text { zebrafish }\end{array}$ & {$[12]$} \\
\hline $\begin{array}{l}\text { ML404 (CID } \\
72199308)\end{array}$ & $\begin{array}{l}\text { Izoxa } \\
\text { zole }\end{array}$ & & ND & $\begin{array}{l}\text { Similar to compounds } \\
\text { disclosed in [12] }\end{array}$ & [79] \\
\hline Ro-68-3400 & $\begin{array}{l}\text { Dihyd } \\
\text { rospir } \\
\text { o[cycl } \\
\text { opent } \\
\text { ane- }\end{array}$ & & VDAC1 & & [87] \\
\hline
\end{tabular}




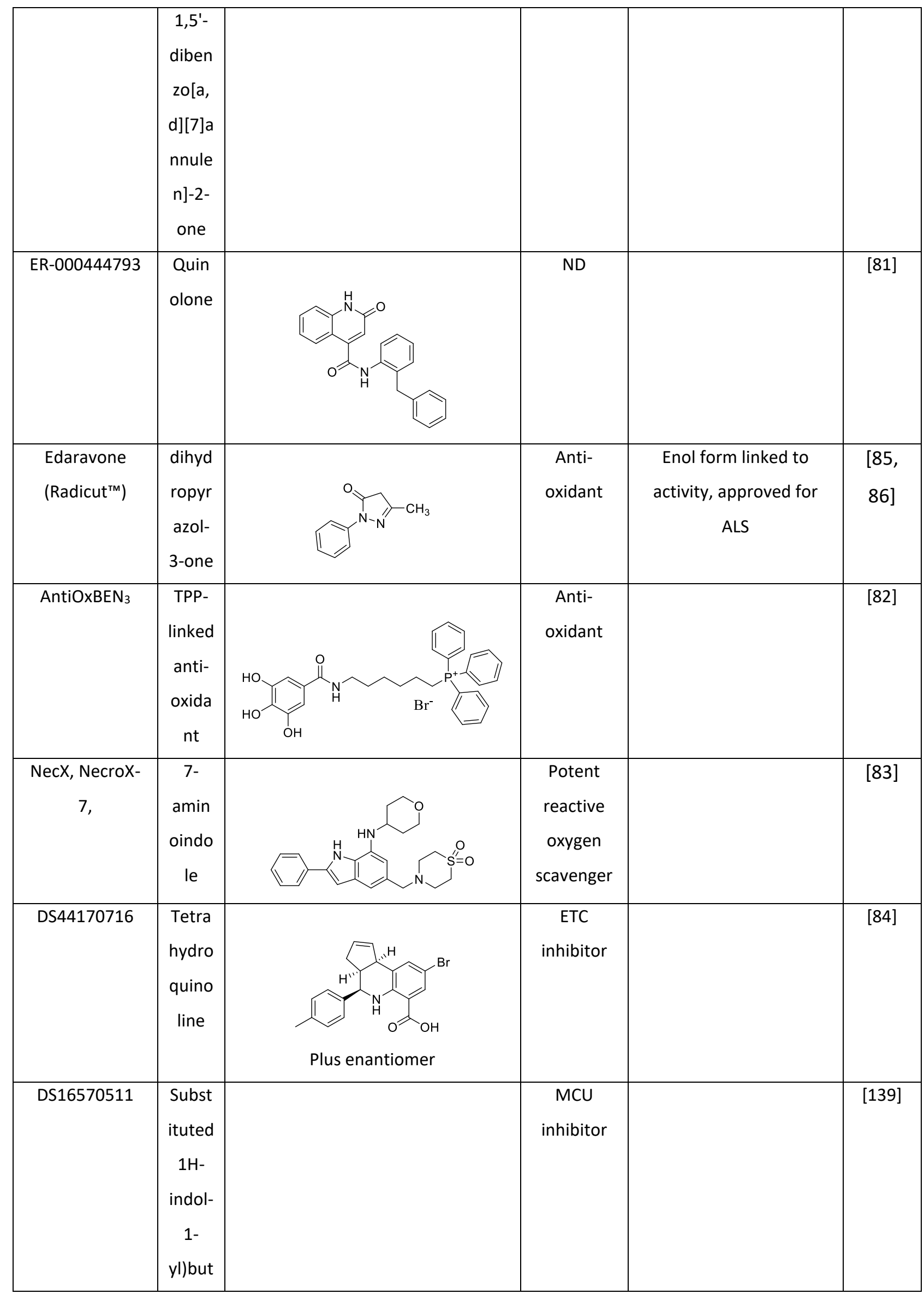




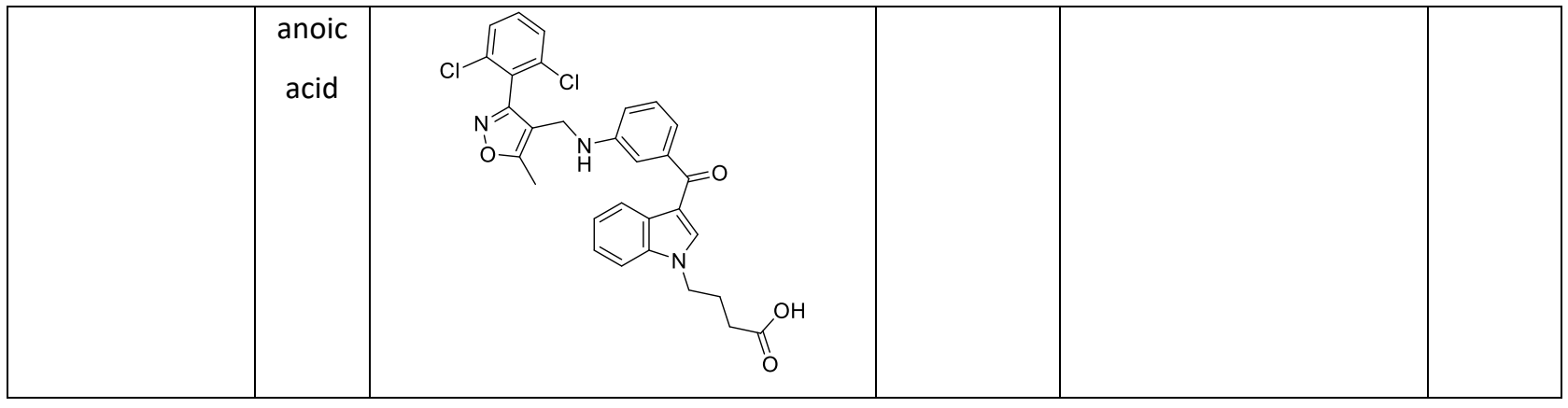

\section{Glossary}

Affinity: Ability of a ligand to bind a biomolecule.

Assay window: Scale of the change in an assay read-out defined by the positive and negative controls (i.e. difference between the mitochondrial calcium uptake capacity in the presence of DMSO (negative control) and CsA (positive control).

Blood brain barrier; BBB: selectively permeable biological membrane separating the central and peripheral systems. BBB is an endothelial cell layer surrounding microvasculature that prevents the movement of molecules from the blood to the brain, protecting the central nervous system from chemicals and micro-organisms.

Bioassay: analytical method designed and optimised to test a biological hypothesis.

DNA-encoded library: a mechanism to massively increase scale of chemical matter screened against a drug target. Compounds are covalently bound to short unique DNA oligonucleotides which are then screened against an immobile protein target. Bound compounds are DNA sequenced allowing elucidation of chemical structure.

Efficacy: ability of a ligand to induce anticipated biological effect.

HTS; High throughput screen: process in drug discovery, often utilising automation and robotics, to simultaneous screen thousands of compounds against a target biomolecule to induce anticipated effect in selected bioassay.

Ischaemia-reperfusion injury: pathology caused subsequent to vascular reperfusion following a period of ischaemia. Reperfusion triggers a cascade of pathophysiological signalling, neutralising acidic $\mathrm{pH}$, restoring mitochondrial transmembrane potential and 
triggering mitochondrial $\mathrm{Ca}^{2+}$ uptake and ATP synthesis. Together, mitochondrial $\mathrm{Ca}^{2+}$ overloading, adenine nucleotide depletion and ROS generation, create the perfect environment for mPTP opening and necrotic cell death.

MCU complex; mitochondrial calcium uniporter complex: protein complex regulating cellular $\mathrm{Ca}^{2+}$ homeostasis and dynamics. The MCU complex comprises a low affinity $\mathrm{Ca}^{2+}$ inner mitochondrial membrane transmembrane channel, the MCU, and regulators MICU1 and MICU2, which together facilitate the electrophoretic movement of $\mathrm{Ca}^{2+}$ from the cytoplasm to the mitochondrial matrix.

Pharmacodynamics: study of 'how a drug acts on an organism' and aims to understand how the biochemical and physiological effects are associated with compound structure.

Pharmacokinetics: study of 'how an organism acts on a drug', typically divided in to understanding drug absorption, distribution, metabolism within the body and excretion and clearance from the body.

Polypharmacology: activity of compounds against multiple biomolecules.

SAR; Structure-activity relationship: Relationship between chemical structure and biological activity. SAR is an iterative cycle of analysis of biological activity following modifications to chemical groups, to allow identification of chemical space responsible for eliciting desired biological effect.

Target Validation (TV): TV necessitates the identification, evaluation and tractability assessment of a 'target' biomolecule. Human biology, genetics, protein expression and biochemical evidence all inform on biomolecule identification. In-house confirmation of literature data and assessment of available reference/tool compounds in disease-relevant bioassays (see Glossary) will confirm tractability and the relationship with a disease phenotype, together contributing to achieving TV.

Z-Prime score: Measure of assay quality using the equation: $Z^{\prime}=1-\frac{3\left(\sigma_{\mathrm{p}}+\sigma_{n}\right)}{\mu_{p}-\mu_{n}}$, taking into account the standard deviation $(\sigma)$ and means $(\mu)$ of the positive $(p)$ and negative $(n)$ controls. A higher Z-prime score suggests greater assay quality. 


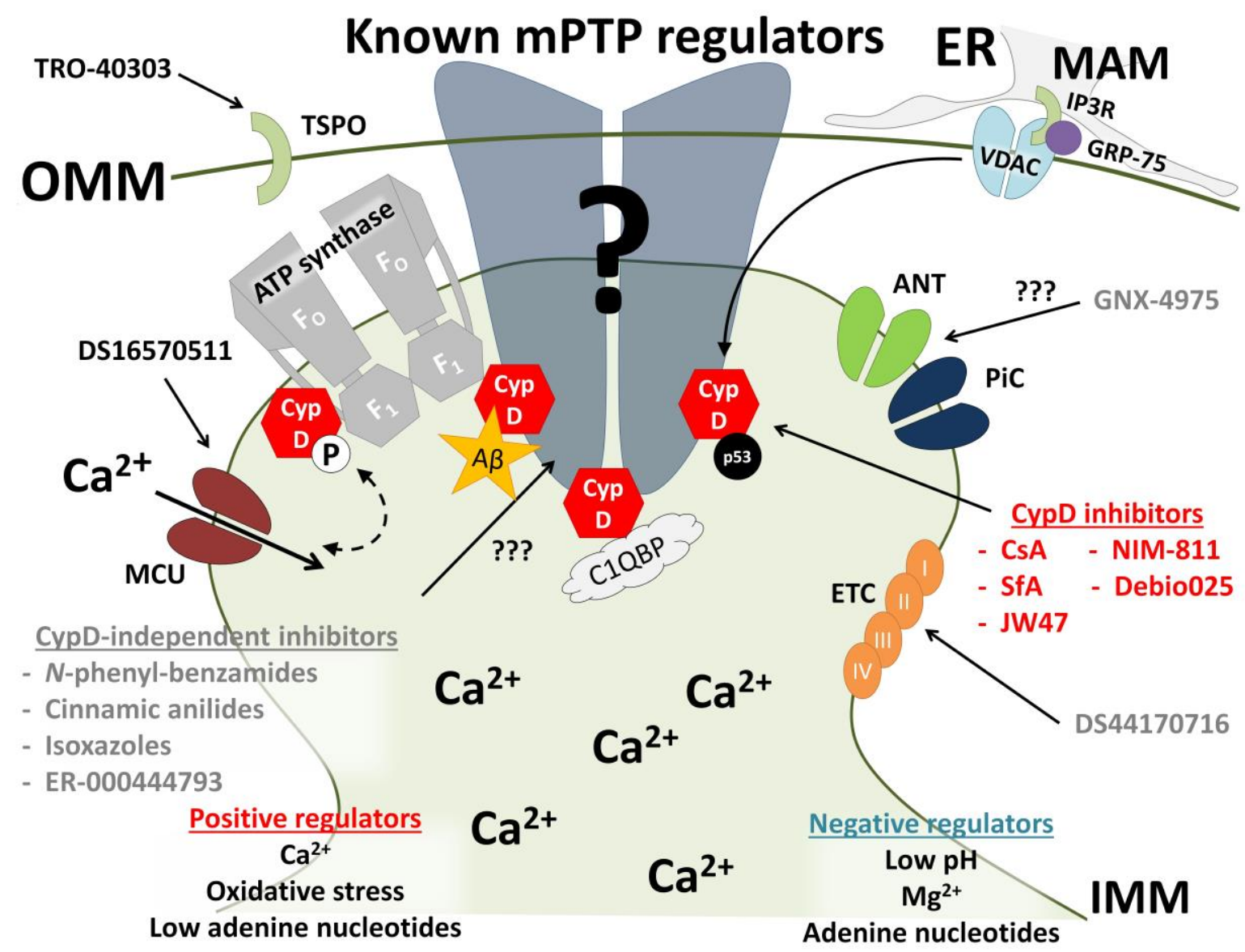

Figure 1. Key Figure 
A

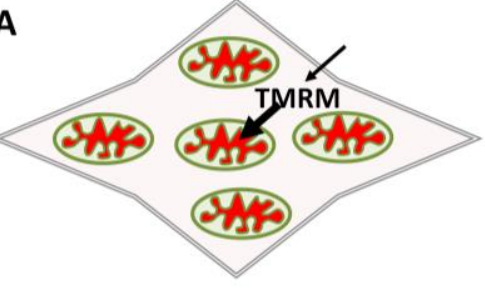

TMRM accumulated in mitochondria (high mitochondrial fluorescence)

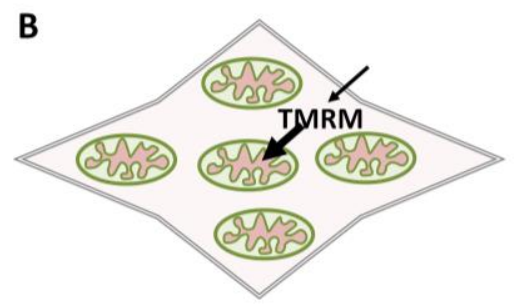

TMRM quenched in mitochondria (low cytosolic fluorescence)
Transmembrane depolarisation Transmembrane depolarisation

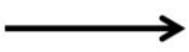

C

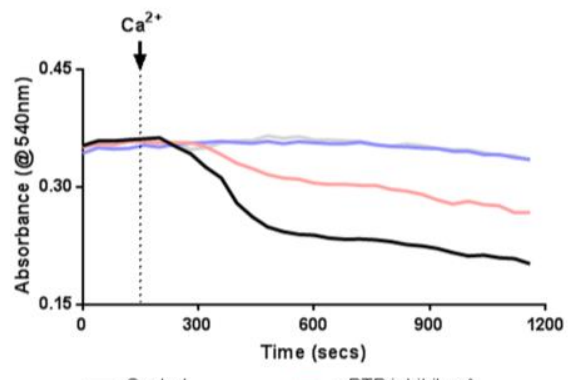

- Control

- mPTP inhibitor $A$

- mPTP inhibitor B - MCU inhibitor/Mito toxin

E

Mitochondrial $\mathrm{Ca}^{2+}$ retention capacity

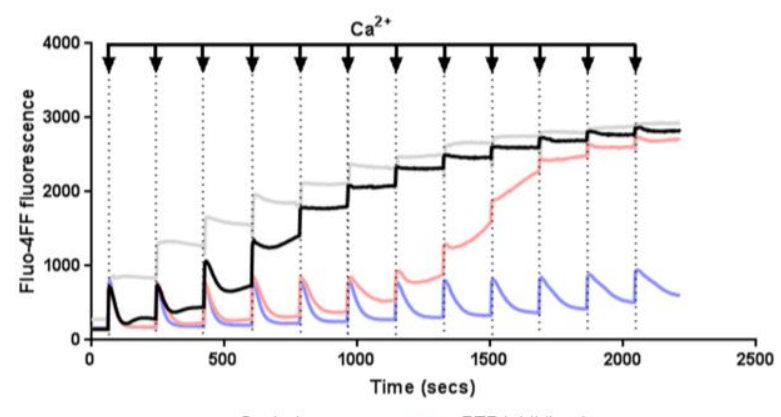

- Control - MPTP inhibitor A

- mPTP inhibitor B - MCU inhibitor/Mito toxin

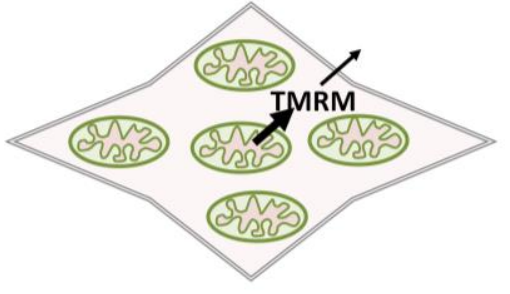

TMRM re-distribution (low mitochondrial fluorescence)

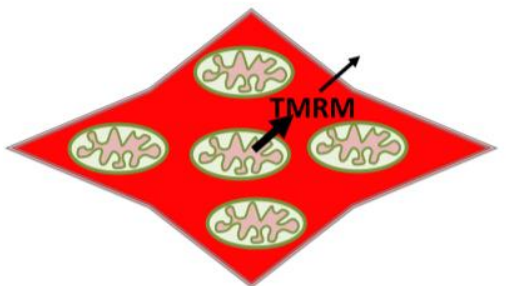

TMRM de-quenched (high cytosolic fluorescence) $\mathrm{Ca}^{2+}$-induced transmembrane depolarisation

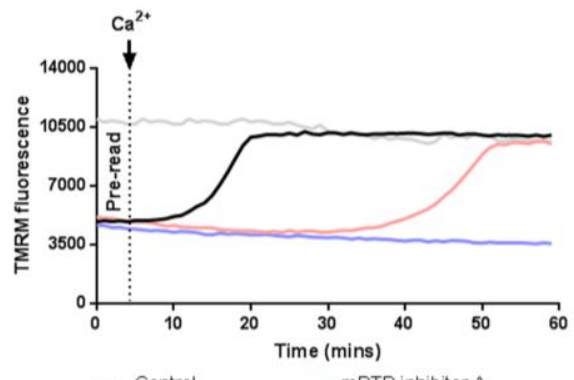

- Control $\quad$ - MPTP inhibitor A

- mPTP inhibitor B - Mito toxin

\section{(Box 2) Figure II:}

